EFI-01-17

hep-th/0106171

\title{
String Theory on AdS Orbifolds
}

\author{
Emil J. Martined and Will McElgin[ \\ Enrico Fermi Inst. and Dept. of Physics \\ University of Chicago \\ 5640 S. Ellis Ave., Chicago, IL 60637, USA
}

\begin{abstract}
We consider worldsheet string theory on $\mathbb{Z}_{N}$ orbifolds of $A d S_{3}$ associated with conical singularities. If the orbifold action includes a similar twist of $S^{3}$, supersymmetry is preserved, and there is a moduli space of vacua arising from blowup modes of the orbifold singularity. We exhibit the spectrum, including the properties of twisted sectors and states obtained by fractional spectral flow. A subalgebra of the spacetime superconformal symmetry remains intact after the $\mathbb{Z}_{N}$ quotient, and serves as the spacetime symmetry algebra of the orbifold.
\end{abstract}

\footnotetext{
${ }^{1}$ e-martinec@uchicago.edu

2 wmcelgin@theory.uchicago.edu
} 


\section{Introduction}

The development of the AdS/CFT correspondence (see [1] for a review, and further references) has given us a wealth of examples of dual realizations of gravity in asymptotically anti-de Sitter space via low energy conformal field theories. A key property exhibited by all of these constructions is holography: the number of degrees of freedom describing physics in a region of space is bounded by the area of that region in Planck units. Unfortunately, the dual description repackages those degrees of freedom in such a way that it is difficult to give a concrete quasilocal description of physics in bulk spacetime, although a number of qualitative checks may be performed, see for example [2, 3, 4, 5, 6, 6]. In particular, one would like to obtain control of a description of black hole formation and evaporation where one sees the horizon, can describe the experience of the infalling observer, etc, in order to finally resolve the puzzles of black hole quantum mechanics.

The present investigation began as an attempt to formulate a situation where one could approach the formation of a black hole state beginning from a description that was string theoretic, local in spacetime, and (as much as possible) perturbative. Our starting point is the description of Giveon, Kutasov and Seiberg [8] of perturbative string theory in $A d S_{3} \times S^{3} \times T^{4}$, which is the near horizon geometry of the bound state of $p$ fundamental strings and $k$ NS fivebranes. The string frame metric on the globally $A d S$ spacetime is

$$
d s^{2}=k\left(-\cosh ^{2} \rho d t^{2}+d \rho^{2}+\sinh ^{2} \rho d \phi^{2}\right)
$$

The relations between the string scale $\ell_{s}$, three-dimensional Planck scale $\ell_{p}$, and $A d S$ curvature scale $\ell$ of the geometry are as follows:

$$
\frac{\ell_{s}}{\ell_{p}}=4 p \sqrt{k} \quad, \quad \frac{\ell}{\ell_{p}}=4 p k \quad, \quad \frac{\ell}{\ell_{s}}=\sqrt{k} .
$$

In addition, the six-dimensional string coupling is $g_{6}^{2}=k / p$. Introducing a pointlike object of mass below the bound for black hole formation into this spacetime creates a conical singularity. This may be described in terms of a global AdS geometry with an angular variable $\tilde{\phi}$ which is identified under $\tilde{\phi} \sim \tilde{\phi}+2 \pi \gamma$. The relation between the deficit angle and the mass in terms of the $A d S$ scale $\ell=\ell_{s} \sqrt{k}$ is

$$
\ell M=-\frac{1}{2} p k \gamma^{2}=-\gamma^{2} \frac{\ell}{8 \ell_{p}},
$$

with $\gamma=1$ corresponding to the global $A d S_{3}$ spacetime, and the limit $\gamma \rightarrow 0$ describing the extremal BTZ black hole (with $M=0$ in our conventions) [10, 11]. The scale $\ell_{p}$ is the Planck length which appears in the low energy gravity action

\footnotetext{
${ }^{3}$ The six-dimensional string coupling is $g_{6}^{2}=g_{s}^{2} \ell_{s}^{4} / V_{4}$, where $V_{4}$ is the volume of $T^{4}$ or K3; there is a duality $g_{6} \rightarrow 1 / g_{6}$, and a condition $V_{4} / \ell_{s}^{4}<p / k$ for the description in terms of fundamental strings to be valid [8, 9].
} 
defined on the quotient spacetime. In worldsheet string theory, we know how to make a conical singularity in the target space geometry - take the orbifold quotient! After laying out in section 2 our conventions for the target space sigma models on $A d S_{3} \equiv S L(2, \mathbb{R})$ and $S^{3} \equiv S U(2)$, in sections 3 , 田 we describe the spectrum of the $\mathbb{Z}_{N}$ orbifold of these WZW models. Supersymmetry requires that one embeds the $\mathbb{Z}_{N}$ such that it acts simultaneously on $A d S_{3}$ and $S^{3}$. The quantization of the $H$ flux through $S^{3}$ then requires that $N$ divides the number of fivebranes $k$. We describe these orbifolds in section 1 . They describe a class of conical deficit spacetimes which one might consider as supersymmetric point objects of mass

$$
\ell M=-\frac{p k}{2 N^{2}}
$$

embedded in $A d S_{3}$. The $N-1$ twisted sectors contribute moduli, the blowup modes and $B$-fluxes of the orbifold singularity, that might be thought of as internal excitations of the object. When $k$ and $N$ are large, these internal excitations might be thought of as precursors of the states of black holes. The properties of the moduli space are briefly discussed in section 6, where we also discuss other worldsheet CFT's related to the orbifold.

An interesting question, which we will not fully resolve, is what the worldsheet orbifold considered here translates to as an operation on the spacetime CFT, which for generic values of its moduli is the sigma model on the moduli space of instantons [20]. The target space of the orbifold is not invariant under $S L(2, \mathbb{R})$ isometries, as it would be if it were a CFT vacuum. Rather it resembles more a nontrivial state in a CFT. We will show that the spacetime charges of the orbifold lead one to identify it as a particular BPS state in the original spacetime CFT. Thus we see that, in this case, distinct worldsheet theories (the $A d S_{3} \times S^{3} \times T^{4}$ sigma model and its orbifold) describe perturbative string theory in the vicinity of different states in the same nonperturbative theory. The state corresponding to the orbifold is obtained from the spacetime CFT vacuum by the application of a vertex operator carrying macroscopic amounts of spacetime energy and angular momentum. We will also see that there are new moduli beyond the moduli space of string theory on $A d S_{3} \times S^{3} \times T^{4}$ arising from the twisted sectors of the orbifold; these might be interpreted as describing a $4(N-1)$ parameter family of states having the same global charges as the orbifold.

The orbifold quotient acts on the $\mathcal{N}=(4,4)$ superVirasoro algebra of the spacetime CFT. Section 7 examines the effect of the orbifold projection on the spacetime superVirasoro symmetries. The superconformal algebra of the theory as described on the covering space, global $A d S_{3}$, consists of a special set of analytic diffeomorphisms of the asymptotic geometry [21, 22], whose Virasoro central charge is $\tilde{c}=6 \tilde{p} k=3 \ell / 2 \tilde{\ell}_{p} ;$ the orbifold projection selects a certain $\mathbb{Z}_{N}$ invariant subalgebra. Here the Planck length in the covering space is given by $\tilde{\ell}_{p}=N \ell_{p}$ since the volume over which the Einstein-Hilbert Lagrangian is integrated is $N$ times larger than that

\footnotetext{
${ }^{4}$ Similar geometries have been considered in [12, 13, 14, 15, 16, 17, 18, 19].
} 
of the quotient spacetime. Equivalently, long strings which wrap $\tilde{p}$ times in the covering space wrap $p=N \tilde{p}$ times in the asymptotically $A d S_{3}$ quotient space. The spacetime orbifold is a representation of this invariant subalgebra rather than the full Virasoro algebra. It turns out that this invariant subalgebra is itself a twisted $\mathcal{N}=(4,4)$ superVirasoro algebra, having central charge $c=N \tilde{c}$. The twisted sector spectrum should consist of representations of the subalgebra which do not lift to the full superVirasoro algebra. The orbifold spacetime is the $-1 / N$ fractional spectral flow of a Ramond vacuum state which has $1 / N$ of the $S U(2) \mathrm{R}$ charge of the Ramond vacuum state of maximal $\mathrm{R}$ charge $c / 12$. The central charge of this superVirasoro algebra is what is expected from considerations of gravity on asymptotically $A d S_{3}$ spacetimes. That is $c=N \tilde{c}=3 \ell / 2 \ell_{p}$ and is the same as the unorbifolded parent theory on global $A d S_{3} \times S^{3} \times T^{4}$.

We also consider briefly a second class of orbifolds which arises if we relax the requirement of supersymmetry; then one can allow the orbifold to act purely on $A d S_{3}$, and there is now no restriction on the allowed values of $N$. Because $N$ can be arbitrarily large, one can come arbitrarily close to the BTZ threshold $M=0$. Nevertheless, the spacetime theory has tachyons in twisted sectors (of string scale mass), indicating that the 'object' is unstable. We propose this background as a useful arena in which to explore the dynamics of tachyon condensation in the closed string sector; the tachyon instability is confined to the region of the orbifold singularity rather than being spread over all of spacetime, hence one might imagine the orbifold theory describes an unstable point in configuration space that then decays into a final state of strings having the same ADM energy. This situation is thus much like the decay of unstable D-branes [23], a topic of some recent interest. A possible obstruction to this interpretation is the nontrivial K-theory charge carried by fractional D-branes sitting at the orbifold singularity; this charge must disappear (perhaps by a RR Higgs mechanism) after tachyon condensation, or else our proposal for the final state after tachyon condensation is incorrect. The mass of the fractional branes will in general be a function of the tachyon condensate, and could vanish at some point; one could indeed then imagine that the further decay of the system involves condensation of branes that screens away the RR charges. Of course, it is also possible that the system is simply unstable, and decays violently, much as in [24] (see 25] for a recent discussion in the context of $A d S$ spacetimes).

Finally, we note that there may be interesting nonperturbative generalizations of our construction. In the moduli space of the bound state of $p$ onebranes and $k$ fivebranes are other perturbative limits [26, 27] for any other charges $p^{\prime}$ and $k^{\prime}$ such that $p^{\prime} k^{\prime}=p k$. Each of these perturbative limits has a GKS-type description (except $p$ or $k$ equal to one), and thus admits an orbifold by $\mathbb{Z}_{N}$ where $N$ divides $k^{\prime}$. It would thus appear that one can orbifold by the cyclic group whose order is any proper divisor of $p k$. In other perturbative limits than the ones where this is naturally defined, the orbifold will appear as an operation that divides by a group larger than would naively be allowed by perturbative string theory, and yet the resulting background must still be consistent. If so, then the set of allowed orbifold 
constructions in string theory would appear to be larger than heretofore known.

\section{The Bosonic WZW model for $S L(2, \mathbb{R})$}

String theory on $A d S_{3}$ may be formulated in terms of the WZW model [28] on the universal cover of the $S L(2, \mathbb{R})$ group manifold. The bosonic WZW action is given by

$$
S[g]=\frac{k}{8 \pi} \int_{\Sigma^{2}} d^{2} \sigma \delta^{\alpha \beta} \operatorname{tr}\left(g^{-1} \partial_{\alpha} g g^{-1} \partial_{\beta} g\right)+\frac{i k}{12 \pi} \int_{M^{3}} \operatorname{tr}(\omega \wedge \omega \wedge \omega) .
$$

Here $M^{3}$ is a three manifold which has the Euclidean worldsheet $\Sigma^{2}$ as its boundary; $\omega=g^{-1} d g$ is the Maurer-Cartan form on $S L(2, \mathbb{R})$. This action can be expressed in the conventions of [29] as the nonlinear sigma model

$$
S[X]=\frac{k}{2 \pi} \int_{\Sigma^{2}} d^{2} z\left(G_{\mu \nu}+B_{\mu \nu}\right) \partial X^{\mu} \bar{\partial} X^{\nu}
$$

where the length scale $\ell$ of $A d S_{3}$ that would appear in $G$ and $B$ has been absorbed into $k$ so that $k=\ell^{2} / \ell_{s}^{2}$. We parameterize $A d S_{3}$ in global 'cylindrical' coordinates $X^{\mu}=(t, \rho, \phi)$. The generators of $S L(2, \mathbb{R})$

$$
\tau^{1}=\frac{i}{2} \sigma^{3} \quad \tau^{2}=\frac{i}{2} \sigma^{1} \quad \tau^{3}=\frac{1}{2} \sigma^{2}
$$

satisfy the algebra

$$
\left[\tau^{a}, \tau^{b}\right]=i \epsilon^{a b}{ }_{c}^{c}
$$

where the metric is $\eta^{a b}=\operatorname{diag}(+1,+1,-1)=-2 \operatorname{tr}\left(\tau^{a} \tau^{b}\right)$ and $\epsilon^{123}=1$. In terms of these conventions, an element of $S L(2, \mathbb{R})$ is given by

$$
g[X]=e^{2 i \theta_{-} \tau^{3}} e^{-2 i \rho \tau^{1}} e^{2 i \theta_{+} \tau^{3}},
$$

where $\theta_{ \pm}=(t \pm \phi) / 2$. This leads to the following forms for $G$ and $B$

$$
\begin{aligned}
G & =-\cosh ^{2} \rho d t^{2}+d \rho^{2}+\sinh ^{2} \rho d \phi^{2} \\
B & =\sinh ^{2} \rho d t \wedge d \phi .
\end{aligned}
$$

Taking $\varepsilon$ to be the right handed (with respect to $(t, \rho, \phi))$ volume form on $A d S_{3}$ this is equivalent to

$$
H=\ell^{2} d B=-\frac{2}{\ell} \varepsilon .
$$

The WZW action is invariant under the transformation

$$
g(z, \bar{z}) \rightarrow \Omega(z) g(z, \bar{z}) \bar{\Omega}(\bar{z})^{-1}
$$


leading to the currents:

$$
\mathcal{J}(z)=\mathcal{J}_{a} \tau^{a}=-\frac{k}{2} \partial g g^{-1} \quad, \quad \overline{\mathcal{J}}(\bar{z})=\overline{\mathcal{J}}_{a} \tau^{a}=-\frac{k}{2} g^{-1} \bar{\partial} g .
$$

A modified $\left(J^{a}, \bar{J}^{a}\right)$ definition of the currents will be used below. Introducing Cartesian coordinates $\left(x^{1}, x^{2}, x^{3}\right)=\sqrt{k}(\rho \cos \phi, \rho \sin \phi, t)$, in the (large $\left.k\right)$ flat space limit the currents become

$$
\mathcal{J}^{a} \approx i \sqrt{k} \partial x^{a} \quad \overline{\mathcal{J}}^{a} \approx i \sqrt{k} \bar{\partial} x^{a}
$$

Consider the behavior of a field $A(z, \bar{z})$ under the infinitesimal transformation

$$
-i \delta g=\epsilon(z) g-g \bar{\epsilon}(\bar{z})
$$

the Ward identity then takes the form

$$
i \delta A(w, \bar{w})=\oint_{w} \frac{d z}{2 \pi i} \epsilon_{a} \mathcal{J}^{a}(z) A(w, \bar{w})+\oint_{w} \frac{d \bar{z}}{2 \pi i} \bar{\epsilon}_{a} \overline{\mathcal{J}}^{a}(\bar{z}) A(w, \bar{w}) .
$$

Under an infinitesimal time translation $\epsilon=\delta t \tau^{3}$ and $\bar{\epsilon}=-\delta t \tau^{3}$, one finds the energy operator

$$
E_{S L(2)}=\mathcal{J}_{0}^{3}+\overline{\mathcal{J}}_{0}^{3}=\oint \frac{d z}{2 \pi i} \mathcal{J}^{3}-\oint \frac{d \bar{z}}{2 \pi i} \overline{\mathcal{J}}^{3}
$$

An infinitesimal rotation $\epsilon=-\delta \phi \tau^{3}$ and $\bar{\epsilon}=-\delta \phi \tau^{3}$ yields the angular momentum

$$
L_{S L(2)}=\mathcal{J}_{0}^{3}-\overline{\mathcal{J}}_{0}^{3}
$$

It turns out that the Ward identity implies that the currents $\left(\mathcal{J}^{a}, \overline{\mathcal{J}}^{a}\right)$ satisfy OPEs with structure constants of opposite sign. This leads to the convention that $\overline{\mathcal{J}}^{+}$ lowers the $\overline{\mathcal{J}}^{3}$ eigenvalue and does not conform to the conventions for monodromies and parafermion representations in the next two sections. This can be remedied while keeping the same form for $E_{S L(2)}$ and $L_{S L(2)}$ in terms of the zero modes by defining the currents as follows

$$
\begin{array}{ll}
J^{3}=\mathcal{J}^{3} & J^{ \pm}=J^{1} \pm i J^{2}=\mathcal{J}^{ \pm} \\
\bar{J}^{3}=\overline{\mathcal{J}}^{3} & \bar{J}^{ \pm}=\bar{J}^{1} \pm i \bar{J}^{2}=\overline{\mathcal{J}}^{\mp} .
\end{array}
$$

The Ward identity then implies the OPEs

$$
\begin{aligned}
J^{a}(z) J^{b}(w) & \sim \frac{(k / 2) \eta^{a b}}{(z-w)^{2}}+\frac{i \epsilon^{a b}{ }_{c} J^{c}(w)}{(z-w)} \\
\bar{J}^{a}(\bar{z}) \bar{J}^{b}(\bar{w}) & \sim \frac{(k / 2) \eta^{a b}}{(\bar{z}-\bar{w})^{2}}+\frac{i \epsilon^{a b}{ }_{c} \bar{J}^{c}(\bar{w})}{(\bar{z}-\bar{w})} .
\end{aligned}
$$




\section{The unflowed $S L(2, \mathbb{R})$ representations}

We briefly review the $S L(2, \mathbb{R})$ representation content as discussed for example in [30]. As discussed in [31], this content does not enumerate all of the primary states of strings on $A d S_{3}$. The additional representations, which can be described by spectral flow of those discussed in [30], and their relation to the spectrum of the orbifold will be discussed in the next section. The "unflowed" current algebra primaries of the $S L(2, \mathbb{R})$ WZW model are arranged into three sectors:

$$
\mathcal{D}_{j}^{+} \times \mathcal{D}_{j}^{+} \quad, \quad \mathcal{D}_{j}^{-} \times \mathcal{D}_{j}^{-} \quad, \quad \mathcal{C}_{j}^{\alpha} \times \mathcal{C}_{j}^{\alpha} .
$$

Here $\mathcal{D}_{j}^{ \pm}$are discrete representations of the universal cover of $S L(2, \mathbb{R})$ which are described as follows:

$$
\mathcal{D}_{j}^{ \pm}=\{|j, m\rangle \mid m= \pm(j+n) \quad ; \quad(n \geq 0) \in \mathbb{Z}\}
$$

The states with $n=0$ satisfy $J_{0}^{\mp}|j, \pm j\rangle=0$. Unitarity requires $j \in \mathbb{R}$ with $0 \leq j<k / 2$. These bounds are further constrained as described in [8, 31] to $1 / 2 \leq j<(k-1) / 2$. The continuous representations $\mathcal{C}_{j}^{\alpha}$ are described as follows:

$$
\mathcal{C}_{j}^{\alpha}=\{|j, m\rangle \mid m=\alpha+n ;(0 \leq \alpha<1) \in \mathbb{R} ; n \in \mathbb{Z}\} .
$$

Unitarity requires $j=1 / 2+i s$ where $s \in \mathbb{R}$. Note that the eigenvalue of the quadratic Casimir is bounded from above for $\mathcal{D}_{j}^{ \pm}$by $-j(j-1) \leq 1 / 4$. This corresponds to the $\ell^{2} \mu^{2}>-1$ Breitenlohner-Freedman bound for the mass $\mu$ appearing in the Klein-Gordon equation on $A d S_{3}$. Conversely for $\mathcal{C}_{j}^{\alpha}$ the quadratic Casimir is bounded from below by $-j(j-1) \geq 1 / 4$; which implies that the continuous representations describe tachyonic excitations. This will not be true of the spectral flow of these representations to be described below.

\section{The Rotational Orbifold}

\subsection{Twist Ground States}

We now consider the orbifold $A d S_{3} / \mathbb{Z}_{N}$ formed by a $2 \pi / N(N \in \mathbb{Z})$ rotation. As can be seen from the parameterization of the group elements given above, a shift $\phi \rightarrow \phi+\alpha$ may be induced as follows:

$$
g(\phi+\alpha)=e^{i \alpha \tau^{3}} g(\phi) e^{-i \alpha \tau^{3}} .
$$

Thus the group field $g(z, \bar{z})$ of the WZW model has the monodromy

$$
g\left(e^{2 \pi i} z, e^{-2 \pi i} \bar{z}\right)=e^{2 \pi i q / N \tau^{3}} g(z, \bar{z}) e^{-2 \pi i q / N \tau^{3}}
$$


in the presence of the vertex operator $\sigma_{q}$ which creates the ground state of the $q \in \mathbb{Z}_{N}$ twisted sector. The holomorphic currents have monodromies

$$
\begin{aligned}
& J^{3}\left(e^{2 \pi i} z\right)=J^{3}(z) \\
& J^{ \pm}\left(e^{2 \pi i} z\right)=e^{ \pm 2 \pi i q / N} J^{ \pm}(z)
\end{aligned}
$$

leading to the mode expansions for the qth twisted sector

$$
\begin{aligned}
J^{3}(z) & =\sum_{n \in \mathbb{Z}} z^{-n-1} J_{n}^{3} \\
J^{ \pm}(z) & =\sum_{n \in \mathbb{Z}} z^{-n-1 \pm q / N} J_{n \mp q / N}^{ \pm} .
\end{aligned}
$$

Similarly, the anti-holomorphic currents have the monodromies

$$
\begin{aligned}
\bar{J}^{3}\left(e^{-2 \pi i} \bar{z}\right) & =\bar{J}^{3}(\bar{z}) \\
\bar{J}^{ \pm}\left(e^{-2 \pi i} \bar{z}\right) & =e^{\mp 2 \pi i q / N} \bar{J}^{ \pm}(\bar{z})
\end{aligned}
$$

and mode expansions

$$
\begin{aligned}
\bar{J}^{3}(\bar{z}) & =\sum_{n \in \mathbb{Z}} \bar{z}^{-n-1} \bar{J}_{n}^{3} \\
\bar{J}^{ \pm}(\bar{z}) & =\sum_{n \in \mathbb{Z}} \bar{z}^{-n-1 \pm q / N} \bar{J}_{n \mp q / N}^{ \pm} .
\end{aligned}
$$

Imposing the condition that $\sigma_{q}$ is a current algebra primary with $E=L=0$ :

$$
J_{\alpha}^{a}\left|\sigma_{q}\right\rangle=0 \quad \forall \alpha \geq 0 \quad \text { and } \quad \bar{J}_{\beta}^{a}\left|\sigma_{q}\right\rangle=0 \quad \forall \beta \geq 0,
$$

the mode algebras implied by the OPEs of the currents may be used to compute the dimension of the twist vertex operators

$$
h\left(\sigma_{q}\right)=\frac{1}{2 \pi i} \oint d z z\left\langle\sigma_{q}|T(z)| \sigma_{q}\right\rangle .
$$

With $T(z)$ given by the Sugawara construction $T(z)=\frac{1}{k-2} \eta_{a b} J^{a} J^{b}(z)$, one finds

$$
h\left(\sigma_{q}\right)=\bar{h}\left(\sigma_{q}\right)=\frac{k}{k-2} \frac{1}{2}(q / N)(1-q / N)
$$

Note that in the flat space limit $k \rightarrow \infty$, the currents become the translation currents (14) with the $\mathbb{Z}_{N}$ monodromies (26). The properties of the twist operators agree with those computed in [32]. 


\section{2 $S L(2, \mathbb{R})$ Parafermions}

A more explicit construction for the twist vertex operators $\sigma_{q}$ can be given in terms of parafermions. We first introduce a parafermion representation for the currents

$$
J^{3}=-\sqrt{k / 2} \partial X \quad, \quad J^{ \pm}=\psi^{ \pm} e^{ \pm \sqrt{2 / k} X} .
$$

And similarly for the anti-holomorphic currents. Here $X$ is a holomorphic field with OPE

$$
X(z) X(w) \sim-\ln (z-w)
$$

The parafermions $\psi^{ \pm}$have OPEs

$$
\psi^{+}(z) \psi^{-}(w) \sim k(z-w)^{-2-2 / k}, \quad \psi^{ \pm}(z) \psi^{ \pm}(w) \sim 0,
$$

which reflect the OPEs for the $S L(2, \mathbb{R})$ currents (20). The current algebra primaries for the $S L(2, \mathbb{R})$ WZW model are given in terms of parafermions by

$$
\Phi_{j m \bar{m}}^{S L(2)}=\Psi_{j m \bar{m}}^{S L(2)} e^{\sqrt{2 / k}(m X+\bar{m} \bar{X})} .
$$

Here $(m, \bar{m})$ are the eigenvalues of the zero modes $\left(J_{0}^{3}, \bar{J}_{0}^{3}\right)$ and $j$ is the spin eigenvalue of $S L(2, \mathbb{R})$. Note that, since we are considering the non-compact covering group of $S L(2, \mathbb{R}), j$ is not quantized. Also note that, due to modular invariance and the fact that the covering space is simply connected, the holomorphic and antiholomorphic Casimirs are equal

$$
\eta_{a b} J_{0}^{a} J_{0}^{b}=\eta_{a b} \bar{J}_{0}^{a} \bar{J}_{0}^{b}=-j(j-1) .
$$

This implies, given details of the representation content, that $m-\bar{m} \in \mathbb{Z}$. Of course this is just a consequence of requiring that the wave function be single valued under a rotation by $2 \pi$. The dimensions of the primary fields are

$$
h\left(\Phi_{j m \bar{m}}^{S L(2)}\right)=\bar{h}\left(\Phi_{j m \bar{m}}^{S L(2)}\right)=\frac{-j(j-1)}{k-2},
$$

hence the dimensions for the parafermions are

$$
h\left(\Psi_{j m \bar{m}}^{S L(2)}\right)=\frac{-j(j-1)}{k-2}+\frac{m^{2}}{k},
$$

and similarly for $\bar{h}$ with $m \rightarrow \bar{m}$.

\section{The parafermion twist operators}

We now describe the $\mathbb{Z}_{N}$ twist operators in terms of the parafermions. It may be verified that, in terms of conformal dimensions and monodromies with the currents, all of the properties of the twist vertex operator are satisfied by

$$
\sigma_{q}=\Psi_{j_{q}, j_{q}, j_{q}}^{S L(2)} \quad \text { where } \quad j_{q}=\frac{k q}{2 N} .
$$


Thus $\sigma_{q}$ is the parafermion for the lowest weight state in $\mathcal{D}_{j_{q}}^{+}$. In particular using (39), as found in (32) above,

$$
h\left(\Psi_{j_{q}, j_{q}, j_{q}}^{S L(2)}\right)=\frac{k j_{q}-2 j_{q}^{2}}{k(k-2)}=\frac{k}{k-2} \frac{1}{2}(q / N)(1-q / N) .
$$

The monodromies with respect to the currents may be verified by looking at the OPEs with the primaries

$$
J^{ \pm}(z) \Phi_{j, m, \bar{m}}^{S L(2)}(w) \sim(m \pm j)(z-w)^{-1} \Phi_{j, m \pm 1, \bar{m}}^{S L(2)}(w)
$$

which implies, through the free OPE (34),

$$
\begin{aligned}
& J^{+}(z) \Psi_{j_{q}, j_{q}, j_{q}}^{S L(2)}(w) \sim(z-w)^{-(1-q / N)} \widehat{O}^{+}(w) \\
& J^{-}(z) \Psi_{j_{q}, j_{q}, j_{q}}^{S L(2)}(w) \sim(z-w)^{-q / N} \widehat{O}^{-}(w)
\end{aligned}
$$

for some vertex operators $\widehat{O}^{ \pm}$. This may be compared with the result that follows from the mode expansions (27) :

$$
\begin{aligned}
& J^{+}(z)\left|\sigma_{q}\right\rangle \sim z^{-(1-q / N)} J_{-q / N}^{+}\left|\sigma_{q}\right\rangle \\
& J^{-}(z)\left|\sigma_{q}\right\rangle \sim z^{-q / N} J_{-(1-q / N)}^{-}\left|\sigma_{q}\right\rangle .
\end{aligned}
$$

Finally, the $J^{3}$ current is regular with respect to both $\sigma_{q}$ and the parafermions.

\subsection{Fractional Spectral Flow}

\section{The integer spectral flow operator}

As described above, for the discrete representations the $S L(2, \mathbb{R})$ spin quantum number $j$ is constrained to the values $1 / 2 \leq j<(k-1) / 2$. This implies, through the mass shell condition, a limit on the dimensions of vertex operators associated with the spaces with which $A d S_{3}$ may be combined to create a critical string theory. Furthermore there is the expectation that strings of arbitrary level number should be permitted in $A d S_{3}$. For instance, a classical description of string propagation in $A d S_{3}$ involves strings that propagate to spatial infinity. These should be associated with a continuous spectrum of physical states. These problems and the problem that the descendants of the above primaries do not lead to a modular invariant partition function were resolved relatively recently in [31, by considering the role of spectral flow in $S L(2, \mathbb{R})$. Spectral flow by $w$ units introduces a shift in the worldsheet quantum numbers

$$
\begin{aligned}
& L_{0} \rightarrow L_{0}-J_{0}^{3} w-\frac{k}{4} w^{2} \\
& J_{0}^{3} \rightarrow J_{0}^{3}+\frac{k}{2} w
\end{aligned}
$$


and similarly for $\bar{L}_{0}, \bar{J}_{0}^{3}$. In the parafermion representation, the operator that implements this spectral flow is given by

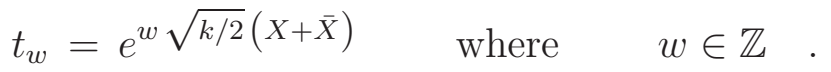

The result of the introduction of these operators is to extend the primary states to include all vertex operators of the form

$$
\Phi_{w j m \bar{m}}^{S L(2)}=\Psi_{j m \bar{m}}^{S L(2)} e^{\sqrt{2 / k}\left(\left(m+\frac{k}{2} w\right) X+\left(\bar{m}+\frac{k}{2} w\right) \bar{X}\right)} .
$$

This leads to the emergence of discrete states of arbitrary level number as well as continuous states that are not tachyonic.

\section{The fractional spectral flow operator}

The spectrum of the orbifold consists of vertex operators which comprise a closed mutually local OPE algebra which includes the twist vertex operators. For the primary states this translates to

$$
\exp \left(i(2 \pi / N) L_{S L(2)}\right)\left|\Phi_{w j m \bar{m}}^{S L(2)}\right\rangle=\left|\Phi_{w j m \bar{m}}^{S L(2)}\right\rangle
$$

which implies the condition $m-\bar{m} \in N \mathbb{Z}$. The twisted sectors consist of states resulting from the OPEs of the surviving untwisted sector states with the twist vertex operators. Consider the most singular term in the OPE of an unflowed lowest weight primary of $\operatorname{spin} j=j_{q}$ with the twist operator of the $(N-q)$ sector

$$
\begin{aligned}
& \Phi_{0 j_{q} j_{q} j_{q}}^{S L(2)}(z, \bar{z}) \sigma_{N-q}(w, \bar{w}) \\
& =e^{q / N} \sqrt{k / 2}(X(z)+\bar{X}(\bar{z})) \sigma_{q}(z, \bar{z}) \sigma_{N-q}(w, \bar{w}) \\
& \sim \frac{C_{q, N-q}}{|z-w|^{4 h\left(\sigma_{q}\right)}} \sigma_{N}(w, \bar{w}) e^{q / N} \sqrt{k / 2}(X(w)+\bar{X}(\bar{w}))+\ldots .
\end{aligned}
$$

Note that $\sigma_{N}$ has dimension zero

$$
h\left(\sigma_{N}\right)=h\left(\Psi_{\frac{k}{2}, \frac{k}{2}, \frac{k}{2}}^{S L(2)}\right)=0 .
$$

It is natural to associate it with the identity operator. We have thus introduced a fractional spectral flow operator into the spectrum

$$
t_{q / N}=e^{q / N} \sqrt{k / 2}(X+\bar{X}) \quad \text { where } \quad q \in \mathbb{Z}_{N}
$$

\footnotetext{
${ }^{5}$ In [33], the spectral flow operators are referred to as twist operators; we prefer to refer to them as spectral flow operators, reserving the term twist operators to refer to the twisted sector vertex operators of the $\mathbb{Z}_{N}$ orbifold.
} 
Fractional spectral flow generates primary states of the orbifold theory that are of the form

$$
\Phi_{p j m \bar{m}}^{S L(2) / \mathbb{Z}_{N}}=\Psi_{j m \bar{m}}^{S L(2)} e^{\sqrt{2 / k}\left(\left(m+\frac{k}{2} \frac{p}{N}\right) X+\left(\bar{m}+\frac{k}{2} \frac{p}{N}\right) \bar{X}\right)} .
$$

Here $m-\bar{m} \in N \mathbb{Z}$ and $p \in \mathbb{Z}$. The twisted sector of the primary is given by $q=N-(p \bmod N)$ as may be verified by the monodromy with the currents. The unflowed primary states, being representations of the zero mode algebra, correspond in the classical limit to geodesics of the geometry. As explained in 31 the geodesics corresponding to the discrete representations of the WZW model are timelike and those of the continuous representations are spacelike. Spectral flow of a geodesic which passes through the origin by $w \in \mathbb{Z}$ stretches the geodesic in the timelike direction, allowing spacelike geodesics corresponding to tachyonic primaries to become timelike, and forms a string worldsheet wrapped $w$ times around the origin as a surface of revolution of the stretched geodesic. The operation of fractional spectral flow introduces strings of this type that wind a fractional number of times, and are closed only by virtue of the orbifold identification. The example of fractional spectral flow on a timelike geodesic for $N=6, w=0$ and $q=2$ is shown in figure 5 .
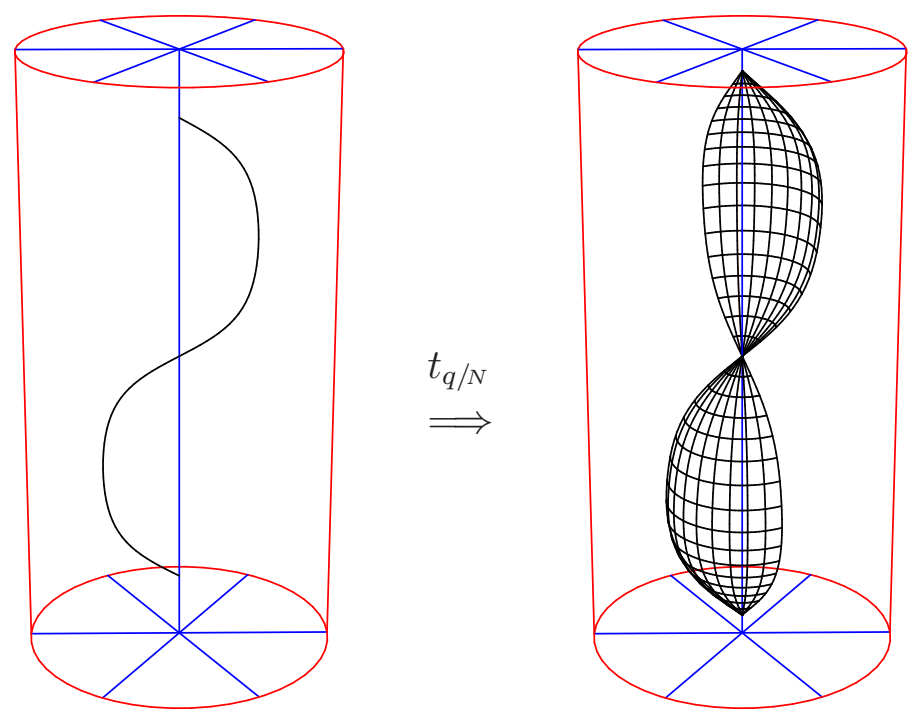

Figure 1: Fractional spectral flow of a timelike geodesic producing a string in the $q=2$ twisted sector for the $N=6$ orbifold.

\section{The $\left(A d S_{3} \times S^{3}\right) / \mathbb{Z}_{N}$ Orbifold}




\subsection{Bosonic Structure}

To construct a critical bosonic string theory $(c=26)$ that has a well defined flat space limit, the WZW model describing string theory on $A d S_{3}$ must be combined with another CFT. Here we will consider some aspects of the bosonic string theory on a $\mathbb{Z}_{N}$ orbifold of $A d S_{3} \times S^{3} \times \mathcal{N}$ associated with a combined rotation of $A d S_{3}$ and $S^{3}$. Here $\mathcal{N}$ is the target space of a bosonic CFT which has central charge $c_{\mathcal{N}}=20$. String theory on $S^{3}$ may be described in terms of a WZW model on the $S U(2)$ group manifold. The description of this WZW model is very similar to that provided above for $S L(2, \mathbb{R})$. One distinction is in the central charges of the bosonic theories

$$
c_{S L(2)}=\frac{3 k_{S L(2)}}{k_{S L(2)}-2} \quad, \quad c_{S U(2)}=\frac{3 k_{S U(2)}}{k_{S U(2)}+2} .
$$

This arises from the difference in the value of the quadratic Casimirs of the adjoint representations of the respective groups. Here we will consider only $c_{S L(2)}+c_{S U(2)}=6$ for the bosonic theory, so that $k_{S L(2)}-2=k_{S U(2)}+2$. The $S U(2)$ group manifold may be parameterized by three Euler angles. One of these angles, which will be denoted $\phi_{S U(2)}$, may be shifted by the transformation

$$
g\left(\phi_{S U(2)}+\alpha\right)=e^{i \alpha \sigma^{3} / 2} g\left(\phi_{S U(2)}\right) e^{-i \alpha \sigma^{3} / 2} .
$$

The orbifold we would like to consider identifies $A d S_{3} \times S^{3}$ as follows

$$
\left(\phi_{S L(2)}, \phi_{S U(2)}\right) \sim\left(\phi_{S L(2)}+2 \pi / N, \phi_{S U(2)}-2 \pi / N\right)
$$

The twisted sector states on this target space may be described by considering the twisted spectra on the rotational orbifolds of the two group manifolds separately and then pairing the $q$ twisted sector of $S L(2, \mathbb{R}) / \mathbb{Z}_{N}$ with the $N-q$ twisted sector of $S U(2) / \mathbb{Z}_{N}$.

\section{The $S U(2) / \mathbb{Z}_{N}$ CFT}

We briefly review the $S U(2) / \mathbb{Z}_{N}$ theory [34. An asymmetric version of this orbifold is described in [35]. When the level $k$ and spin $j$ appear in this paragraph they correspond to $k_{S U(2)}$ and $j_{S U(2)}$. The current algebra primaries of the $S U(2) \mathrm{WZW}$ model are expressed in terms of parafermions as

$$
\Phi_{j m \bar{m}}^{S U(2)}=\Psi_{j m \bar{m}}^{S U(2)} e^{i \sqrt{2 / k}(m Y+\bar{m} \bar{Y})} .
$$

Here $Y$ is a free boson associated with the holomorphic current $J_{S U(2)}^{3}$ :

$$
J_{S U(2)}^{3}=i \sqrt{k / 2} \partial Y \text {. }
$$

And similary for $\bar{Y}$. As for the $S L(2, \mathbb{R})$ case, the holomorphic and antiholomorhic Casimirs are equal. The dimension of the primaries, as computed using the Sugawara 
stress tensor $T(z)=\frac{1}{k+2} \delta_{a b} J^{a} J^{b}(z)$, is given by $h\left(\Phi_{j m \bar{m}}^{S U(2)}\right)=\frac{j(j+1)}{k+2}$; subtracting the contribution of the free boson yields the dimensions of the parafermions

$$
h\left(\Psi_{j m \bar{m}}^{S U(2)}\right)=\frac{j(j+1)}{k+2}-\frac{m^{2}}{k} .
$$

And similarly for $\bar{h}$ with $m \rightarrow \bar{m}$. The twist vertex operators of the $S U(2) / \mathbb{Z}_{N}$ orbifold are given by the lowest weight state in the $\mathcal{D}_{j_{q}}^{S U(2)}$ representation. That is

$$
\sigma_{q}^{S U(2)}=\Psi_{j_{q},-j_{q},-j_{q}}^{S U(2)} \quad \text { where } \quad j_{q}=\frac{k q}{2 N}
$$

Note that the $j=j_{q}$ representation exists since the level $k$ of the orbifold theory is restricted to $k_{S U(2)} \in N \mathbb{Z} ; S U(2) / \mathbb{Z}_{N}$ is a compact manifold with a volume that is $1 / N$ of that of $S U(2)$, and the $H$ flux threading it must be an integer. Also note that, as for the $S L(2, \mathbb{R}) / \mathbb{Z}_{N}$ orbifold, $m-\bar{m} \in N \mathbb{Z}$. The dimensions of the twist operators are thus given by

$$
h\left(\sigma_{q}^{S U(2)}\right)=\frac{k j_{q}-2 j_{q}^{2}}{k(k+2)}=\frac{k}{k+2} \frac{1}{2}(q / N)(1-q / N)
$$

which, as for $S L(2, \mathbb{R}) / \mathbb{Z}_{N}$ above, agrees with the calculation [32] in the $k \rightarrow \infty$ flat space limit. The twist operators imply the existence of fractional spectral flow operators

$$
t_{q / N}^{S U(2)}=e^{i q / N} \sqrt{k / 2}(Y+\bar{Y}) \quad \text { where } \quad q \in \mathbb{Z}_{N} .
$$

However there is no independent spectral flow quantum number $w$ characterizing the representations of the (compact) $S U(2)$ WZW model, since integer spectral flow maps the current algebra representations into themselves.

\subsection{The Supersymmetric Orbifold}

\section{The supersymmetric WZW model}

We now consider the superstring on the orbifold $\left(A d S_{3} \times S^{3}\right) / \mathbb{Z}_{N} \times \mathcal{N}$. To form a critical $c=15$ theory, the $\mathrm{CFT}$ on $\mathcal{N}$ is required to have $c_{\mathcal{N}}=6$. Only the case $\mathcal{N}=T^{4}$ will be described in detail; the generalization is straightforward. The OPEs and Sugawara stress tensor are described in what follows for both the $S U(2)$ and $S L(2, \mathbb{R})$ supersymmetric level $k$ WZW model [36] with the Killing metric $g_{a b}$ and quadratic Casimir $Q$. The WZW supercurrent $C^{a}$ is expressed in terms of the total current $J^{a}$ and the fermions $\psi^{a}$ as

$$
C^{a}=\psi^{a}+\theta J^{a}
$$

Here $\theta$ is the holomorphic worldsheet Grassmann coordinate. 
$J^{a}$ and $\psi^{a}$ satisfy the OPEs :

$$
\begin{aligned}
J^{a}(z) J^{b}(w) & \sim \frac{(k / 2) g^{a b}}{(z-w)^{2}}+\frac{i \epsilon^{a b}{ }_{c} J^{c}(w)}{(z-w)} \\
J^{a}(z) \psi^{b}(w) & \sim \frac{i \epsilon^{a b}{ }_{c} \psi^{c}(w)}{(z-w)} \\
\psi^{a}(z) \psi^{b}(w) & \sim \frac{(k / 2) g^{a b}}{(z-w)}
\end{aligned}
$$

Subtracting the contribution to $J^{a}$ which comes from the fermionic piece of the SUSY WZW action produces the bosonic current

$$
j^{a}=J^{a}+(i / k) \epsilon_{b c}^{a} \psi^{b} \psi^{c},
$$

which leads to the OPEs

$$
\begin{aligned}
& j^{a}(z) j^{b}(w) \sim \frac{(\tilde{k} / 2) g^{a b}}{(z-w)^{2}}+\frac{i \epsilon^{a b}{ }_{c} j^{c}(w)}{(z-w)} \\
& j^{a}(z) \psi^{b}(w) \sim 0 .
\end{aligned}
$$

Where $\tilde{k}=k-Q$. The stress tensor may be obtained as usual:

$$
T=\frac{1}{\tilde{k}+Q} j^{a} j^{b} g_{a b}-\frac{1}{k} \psi^{a} \partial \psi^{b} g_{a b}
$$

and the Virasoro central charge is

$$
c=\frac{3 \tilde{k}}{\tilde{k}+Q}+\frac{3}{2} .
$$

The Virasoro supercurrent is given by

$$
G=\frac{2}{k}\left(g_{a b} \psi^{a} j^{b}-\frac{i}{3 k} \epsilon_{a b c} \psi^{a} \psi^{b} \psi^{c}\right) .
$$

The superstring on $A d S_{3} \times S^{3} \times T^{4}$

We briefly review here the description of the superstring on $A d S_{3} \times S^{3} \times T^{4}$ as it appears in [8, 35]. The fermions and the total and bosonic currents associated with the $\widehat{S L}(2, \mathbb{R})$ current algebra will be denoted by $\left(\psi^{A}, J^{A}, j^{A}\right)$ respectively. For $\widehat{S U}(2)$ they will be denoted by $\left(\chi^{a}, K^{a}, k^{a}\right)$. The (canonically normalized) $\widehat{U}(1)^{4}$ fermions and current will be denoted $\lambda^{j}$ and $i \partial F^{j}$. Note that the levels of the associated bosonic WZW current algebras are shifted as described above. That is, for the superstring

$$
c_{S L(2)}=\frac{3\left(k_{S L(2)}+2\right)}{k_{S L(2)}}+\frac{3}{2} \quad, \quad c_{S U(2)}=\frac{3\left(k_{S U(2)}-2\right)}{k_{S U(2)}}+\frac{3}{2} .
$$


The condition $c=15$ then leads to $k=k_{S L(2)}=k_{S U(2)}$. The ten fermions may be written in terms of five canonically normalized free bosons $H_{I}$ where $I \in(1, \ldots, 5)$ as follows

$$
\begin{aligned}
i \partial H_{1} & =-i \frac{2}{k} \psi^{1} \psi^{2}=J^{3}-j^{3} \\
i \partial H_{2} & =-i \frac{2}{k} \chi^{1} \chi^{2}=K^{3}-k^{3} \\
i \partial H_{3} & =-\frac{2}{k} \psi^{3} \chi^{3} \\
i \partial H_{4} & =-i \lambda^{1} \lambda^{2} \\
i \partial H_{5} & =-i \lambda^{3} \lambda^{4} .
\end{aligned}
$$

The spacetime supercharges are constructed as in [37] :

$$
Q_{\alpha}=\oint \frac{d z}{2 \pi i} e^{-\varphi / 2} S_{\alpha}(z),
$$

where $\varphi$ is a boson of the $\beta, \gamma$ superghost system and

$$
S_{\alpha}=\exp \left(\frac{i}{2} \epsilon_{I} H_{I}\right)
$$

is one of the 32 spin fields possible before the imposition of the GSO projection and the condition of BRST invariance. Here $\alpha=\left(\epsilon_{1} \ldots \epsilon_{5}\right)$ where $\epsilon_{I}= \pm 1$. The GSO projection amounts to imposing

$$
\prod_{I=1}^{5} \epsilon_{I}=1
$$

BRST invariance is not guaranteed due to the presence of the term cubic in the fermions in $G(z)$ (68). The cancellation of the term of order $z^{-3 / 2}$ in the $G(z) S_{\alpha}(0)$ OPE leads to the condition

$$
\prod_{I=1}^{3} \epsilon_{I}=1
$$

Thus there are 8 'left-moving' supercharges on $A d S_{3} \times S^{3} \times T^{4}$ as compared to 16 in flat space (and 8 more from the right-movers).

\section{Massless and spacetime chiral states}

As shown explicity in $\mathbb{8}$, the worldsheet $\widehat{S L}(2, \mathbb{R}) \times \widehat{S U}(2) \times \widehat{U}(1)^{4}$ current algebra is associated with an $\mathcal{N}=4$ superconformal algebra in spacetime. The global modes of the spacetime Virasoro and current algebras are just the charges of the associated total worldsheet currents. Thus for $\widehat{S L}(2, \mathbb{R})$ and $\widehat{S U}(2)$ :

$$
\mathcal{L}_{0}=\oint \frac{d z}{2 \pi i} J^{3}(z) \quad \mathcal{L}_{ \pm}=\oint \frac{d z}{2 \pi i} J^{\mp}(z)
$$


and

$$
\mathcal{T}_{0}^{a}=\oint \frac{d z}{2 \pi i} K^{a}(z)
$$

Chiral primary states of this spacetime algebra were described in [8] [35] in terms of the unflowed worldsheet current algebra primaries as follows. The worldsheet primaries of the WZW supercurrents are given by the primaries of the bosonic (level $(k+2)$ for $S L(2, \mathbb{R})$ and $(k-2)$ for $S U(2))$ WZW model

$$
\Phi_{j m \bar{m}}^{S L(2)} \Phi_{j^{\prime} m^{\prime} \bar{m}^{\prime}}^{S U(2)} e^{i \mathbf{p} \cdot F+i \overline{\mathbf{p}} \cdot \bar{F}}
$$

Here $(\mathbf{p}, \overline{\mathbf{p}})$ is a vector in an even, self-dual Narain lattice $\Gamma^{4,4}$. Physical states consist of bosonic current algebra descendants and fermionic excitations of these primaries which are in the BRST cohomology. Suppressing the anti-holomorphic quantum numbers, the $\widehat{S L}(2, \mathbb{R})$ primary states satisfy [ 8

$$
\left[\mathcal{L}_{n}, \Phi_{j, m}^{S L(2)}\right]=(n(j-1)-m) \Phi_{j, m+n}^{S L(2)}
$$

where $\mathcal{L}_{n}$ are generators of the spacetime Virasoro algebra. Thus these primaries can be seen to be modes of an operator with spacetime scaling dimension $h=j$. Unitarity of the spacetime superconformal algebra requires that $h \geq j_{S U(2)}$, where $j_{S U(2)}$ is the spin associated with the spacetime $\widehat{S U}(2)$ current algebra $\mathcal{T}_{n}^{a}$. This is, of course, also the spin of the total worldsheet $\widehat{S U}(2)$ current $K^{a}$. The chiral primary operators in the NS sector saturate this bound and satisfy $\mathbf{p}=0$ and $\mathbf{N}=1 / 2$, where $\mathbf{N}$ is the total level of the oscillator excitations. Physical states which are spacetime chiral primaries correspond to massless excitations as can be seen from the NS sector mass-shell condition

$$
\frac{-j(j-1)}{k}+\frac{j^{\prime}\left(j^{\prime}+1\right)}{k}+\frac{\mathbf{p} \cdot \mathbf{p}}{2}+\mathbf{N}=\frac{1}{2} .
$$

This implies $j^{\prime}=j-1$. There are eight massless physical NS states for a given $j$ as well as $m, m^{\prime}$ (which are suppressed)

$$
\begin{aligned}
\mathcal{V}_{j}^{i} & =e^{-\varphi} \lambda^{i} \Phi_{j}^{S L(2)} \Phi_{j^{\prime}}^{S U(2)} \\
\mathcal{W}_{j}^{ \pm} & =e^{-\varphi}\left[\psi \Phi_{j}^{S L(2)}\right]_{j \pm 1} \Phi_{j^{\prime}}^{S U(2)} \\
\mathcal{X}_{j}^{ \pm} & =e^{-\varphi} \Phi_{j}^{S L(2)}\left[\chi \Phi_{j^{\prime}}^{S U(2)}\right]_{j^{\prime} \pm 1}
\end{aligned}
$$

Here the quantum numbers outside the brackets refer to $j_{S L(2)}$ and $j_{S U(2)}$, the spins associated with the quadratic Casimirs of the total currents $J^{a}$ and $K^{a}$ respectively.

\footnotetext{
${ }^{6}$ Note that a different convention is used here for the $S L(2, \mathbb{R}) j$ quantum number than is used in $[8,35$.
} 
Details of the construction of these states may be found in 35. The additional two states

$$
\begin{aligned}
\mathcal{W}_{j}^{0} & =e^{-\varphi}\left[\psi \Phi_{j}^{S L(2)}\right]_{j} \Phi_{j^{\prime}}^{S U(2)} \\
\mathcal{X}_{j}^{0} & =e^{-\varphi} \Phi_{j}^{S L(2)}\left[\chi \Phi_{j^{\prime}}^{S U(2)}\right]_{j^{\prime}}
\end{aligned}
$$

are not in the BRST cohomology. Of these massless states only $\mathcal{W}_{j}^{-}$and $\mathcal{X}_{j}^{+}$are spacetime chiral primaries, that is are modes of spacetime operators which satisfy $h=j_{S L(2)}=j_{S U(2)}$. Ramond sector states are found by applying the spacetime supercharges to these NS states [37].

In all of these vertex operators, the current algebra primaries $\Phi_{j^{\prime} m^{\prime} \bar{m}^{\prime}}^{S U(2)}$ and $\Phi_{j m \bar{m}}^{S L(2)}$ span the space of wavefunctions on $A d S_{3} \times S^{3}$ for spins less than $k / 2$, while the fermions $\psi, \chi, \lambda$ carry the polarizations of the various supergravity modes.

\section{Superparafermions}

Due to the contribution of the fermions to the total currents $J^{a}$ and $K^{a}$ of $S L(2, \mathbb{R})$ and $S U(2)$, respectively, it is convenient to write the current algebra primaries in terms of superparafermions times exponentials of the bosons related to the total currents $J^{3}$ and $K^{3}$. For example for the $\widehat{S U}(2)$ superparafermions

$$
\Phi_{j m \bar{m}}^{S U(2)}=\widehat{\Psi}_{j m \bar{m}}^{S U(2)} \exp \left[i \sqrt{\frac{2}{k}}(m \mathcal{Y}+\bar{m} \overline{\mathcal{Y}})\right] .
$$

Where we have bosonized the total current as $K^{3}=i \sqrt{k / 2} \partial \mathcal{Y}$. The bosonic current $k^{3}=i \sqrt{\tilde{k} / 2} \partial Y$ of the parafermion construction of (57) (referred to as $J_{S U(2)}^{3}$ there) and the boson $\mathrm{H}_{2}$ of $(70)$ are then rewritten in terms of $\mathcal{Y}$ and another boson $\mathcal{H}_{2}$ (both canonically normalized) via円

$$
\begin{aligned}
Y & =\sqrt{\frac{\tilde{k}}{k}} \mathcal{Y}-\sqrt{\frac{2}{k}} \mathcal{H}_{2} \\
H_{2} & =\sqrt{\frac{\tilde{k}}{k}} \mathcal{H}_{2}+\sqrt{\frac{2}{k}} \mathcal{Y} .
\end{aligned}
$$

One also has the following relation between the level $k$ superparafermions and the parafermions of the bosonic $S U(2)$ level $k-2$ WZW model

$$
\widehat{\Psi}_{j m \bar{m}}^{S U(2)}=\Psi_{j m \bar{m}}^{S U(2)} \exp \left[-i \sqrt{\frac{4}{\tilde{k} k}}\left(m \mathcal{H}_{2}+\bar{m} \overline{\mathcal{H}}_{2}\right)\right] .
$$

The bosonic currents and associated fermions are given by

$$
\begin{aligned}
k^{1} \pm i k^{2} & =\psi^{ \pm} \exp \left[ \pm i \sqrt{\frac{2}{k}}\left(\mathcal{Y}-\sqrt{\frac{2}{\tilde{k}}} \mathcal{H}_{2}\right)\right] \\
\chi^{1} \pm i \chi^{2} & =\sqrt{k} \exp \left[ \pm i\left(\sqrt{\frac{2}{k}} \mathcal{Y}+\sqrt{\frac{\tilde{k}}{k}} \mathcal{H}_{2}\right)\right]
\end{aligned}
$$

\footnotetext{
${ }^{7}$ Recall our notation $\tilde{k}=k-Q$. Thus, for $S U(2), Q=2$ and $\tilde{k}=k-2$; while for $S L(2, \mathbb{R})$, $Q=-2$ and $\tilde{k}=k+2$.
} 
and the supercurrent is written as

$$
\sqrt{k} G=\psi^{+} e^{-i \sqrt{k / \tilde{k}} \mathcal{H}_{2}}+\psi^{-} e^{+i \sqrt{k / \tilde{k}} \mathcal{H}_{2}}+\sqrt{2} \chi^{3} i \partial \mathcal{Y}
$$

Similarly one may write $X$ and $H_{1}$ of the $S L(2, \mathbb{R})$ supersymmetric WZW model in terms of bosons $\mathcal{X}, \mathcal{H}_{1}$ of the analogous superparafermionic construction for $S L(2, \mathbb{R})$. The advantage of the superparafermion description is that it makes manifest the $\mathbb{Z}_{k}$ symmetry of the supersymmetric $S U(2)$ WZW model, which acts only on the boson $\mathcal{Y}$ which bosonizes the (total) current $J_{S U(2)}^{3}$ (whereas the bosonic parafermions have a $\mathbb{Z}_{\tilde{k}}$ symmetry acting on $Y$ ). Furthermore the superparafermion operators are by construction primary fields of the superVirasoro algebra. Thus the twist operators for the superstring on the orbifold $\left(A d S_{3} \times S^{3}\right) / \mathbb{Z}_{N} \times \mathcal{N}$ (where $N$ divides $k$ ) will be written in terms of the superparafermions $\widehat{\Psi}_{j m \bar{m}}^{S L(2)}$ and $\widehat{\Psi}_{j m \bar{m}}^{S U(2)}$.

\section{The $\left(A d S_{3} \times S^{3}\right) / \mathbb{Z}_{N} \times T^{4}$ orbifold}

We now consider the superstring on the orbifold $\left(A d S_{3} \times S^{3}\right) / \mathbb{Z}_{N} \times T^{4}$. The twist vertex operators for the superstring which have the proper monodromy with respect to the currents and have a single valued OPE with the worldsheet supercurrent $G(z)$ are constructed as follows. The twist operators on $S L(2, \mathbb{R}) / \mathbb{Z}_{N}$ are given by the superparafermions

$$
\Omega_{q}^{S L(2)}=\hat{\Psi}_{j_{q}, j_{q}, j_{q}}^{S L(2)}=\Psi_{j_{q}, j_{q}, j_{q}}^{S L(2)} \exp \left[i \sqrt{\frac{k}{k}} \frac{q}{N}\left(\mathcal{H}_{1}+\overline{\mathcal{H}}_{1}\right)\right]
$$

here again $j_{q}=k q / 2 N$, and $\tilde{k}=k-Q$. Similarly the twist operators on $S U(2) / \mathbb{Z}_{N}$ are given by

$$
\Omega_{q}^{S U(2)}=\hat{\Psi}_{j_{q}, j_{q}, j_{q}}^{S U(2)}=\Psi_{j_{q}, j_{q}, j_{q}}^{S U(2)} \exp \left[i \sqrt{\frac{k}{\tilde{k}}} \frac{q}{N}\left(\mathcal{H}_{2}+\overline{\mathcal{H}}_{2}\right)\right] .
$$

The dimension of both the superparafermions is $h\left(\Omega_{q}\right)=q / 2 N$; thus the twist operators on the $\left(A d S_{3} \times S^{3}\right) / \mathbb{Z}_{N} \times T^{4}$ orbifold are

$$
\Omega_{q}^{\text {orb }}=\Omega_{q}^{S L(2)} \Omega_{N-q}^{S U(2)} \quad, \quad q=1, \ldots, N-1 ;
$$

each of these states has vanishing spacetime energy $\mathcal{L}_{0}$ and $S^{3}$ angular momentum $\mathcal{T}_{0}^{3}$. The condition for primary fields to be invariant under the action of the orbifold identification

$$
\exp \left[i(2 \pi / N)\left(L_{S L(2)}-L_{S U(2)}\right)\right]\left|\Phi_{j m \bar{m}}^{S L(2)} \Phi_{j^{\prime} m^{\prime} \bar{m}^{\prime}}^{S U(2)}\right\rangle=\left|\Phi_{j m \bar{m}}^{S L(2)} \Phi_{j^{\prime} m^{\prime} \bar{m}^{\prime}}^{S U(2)}\right\rangle
$$

implies the condition $(m-\bar{m})-\left(m^{\prime}-\bar{m}^{\prime}\right) \in N \mathbb{Z}$. Here the generators of rotation are given by

$$
L_{S L(2)}=J_{0}^{3}-\bar{J}_{0}^{3} \quad L_{S U(2)}=K_{0}^{3}-\bar{K}_{0}^{3} .
$$

\footnotetext{
${ }^{8}$ Note however the shift in notation - in the supersymmetric case the order of the cyclic orbifold group $N$ divides the level $k$ of the total current, rather than the bosonic level $\tilde{k}$. Hence one must suitably modify the formulae (41), 600) for the parafermion dimensions.
} 
Consider the OPE of the twist vertex operators with the spin fields used to construct the spacetime supercharges

$$
\Omega_{q}^{O R B}(z) S_{\alpha}(0) \sim z^{\epsilon_{2} / 2} z^{q / N\left(\epsilon_{1}-\epsilon_{2}\right) / 2}: \Omega_{q}^{O R B}(z) S_{\alpha}(0): \quad ;
$$

this implies $\epsilon_{1}=\epsilon_{2}$ and thus, from (74) above, $\epsilon_{3}=1$. Thus only 4 'left-moving' supercharges survive the orbifold projection out of the original 8 on $A d S_{3} \times S^{3} \times T^{4}$ (and 4 more from the right-movers). The associated spin fields can be indexed by the $i \partial H_{1}$ and $i \partial H_{4}$ charges

$$
S_{\epsilon_{1} \epsilon_{4}}=e^{\frac{i}{2}\left(\epsilon_{1}\left(H_{1}+H_{2}\right)+H_{3}+\epsilon_{4}\left(H_{4}+H_{5}\right)\right)} .
$$

Note that the twist operators (89) commute with half of the supersymmetry generators, thus the corresponding states (and their fractional spectral flows) are BPS.

\section{The supersymmetric (fractional) spectral flow operator}

The integer spectral flow operator introduced in 31] was extended to the superstring in 33. Express the total currents $J^{3}$ and $K^{3}$ in terms of bosons $\mathcal{X}$ and $\mathcal{Y}$

$$
J^{3}=-\sqrt{k / 2} \partial \mathcal{X} \quad K^{3}=i \sqrt{k / 2} \partial \mathcal{Y}
$$

The holomorphic part of the integer spectral flow operator for the superstring is

$$
t_{w}=e^{w \sqrt{k / 2}(\mathcal{X}+i \mathcal{Y})}
$$

Note that $h\left(t_{w}\right)=0$ and that $t_{w}$ is mutually local with respect to the spacetime supercharges. Integer spectral flow $\mathcal{O}_{\mathrm{BPS}} \rightarrow t_{w} \mathcal{O}_{\mathrm{BPS}}, w \in \mathbb{Z}$, extends the range of BPS operators (80) beyond the window $\frac{1}{2} \leq j<\frac{k-1}{2}$ of discrete series highest weight representations. The orbifold again admits fractional spectral flow under (95) with $w=p / N, p \in \mathbb{Z}$, describing oscillating strings of the sort depicted in figure 1 . The BPS single particle spectrum of the orbifold thus consists of the surviving untwisted sector supergravity states $(80)$ (i.e. those with $\left.(m-\bar{m})-\left(m^{\prime}-\bar{m}^{\prime}\right) \in N \mathbb{Z}\right)$, together with their fractional spectral flows by (95) (with $w=p / N$ ); furthermore there are the twist ground states (89) and their fractional spectral flows.

The continuous representations $\mathcal{C}_{j}^{\alpha}$ of $S L(2, \mathbb{R})$ spin $j=\frac{1}{2}+i s$ appear in the spectrum of the $A d S_{3} \times S^{3} \times T^{4}$ theory in nonzero spectral flow sectors, where they describe a continuum of long strings moving in or out from the boundary of $A d S_{3}$ with radial momentum $s$. One might think that the $\mathbb{Z}_{N}$ orbifold identification lowers the threshold of the continuum of long string states by a factor of order $N$, since a long string need only wind part of the way around the $A d S_{3}$ angular direction.

From the analysis of [31, 33], a continuous representation of spin $j=\frac{1}{2}+i s$ can be spectrally flowed $w$ units and then used to dress a physical vertex operator $e^{-\varphi} \Phi_{j m}^{w} \mathcal{O}_{\text {int }}$ (where $\mathcal{O}_{\text {int }}$ is a vertex operator in the remaining $S^{3} \times T^{4}$ sigma model), for which the mass shell condition is

$$
-\frac{j(j-1)}{k}-w m-\frac{k}{4} w^{2}+h_{\mathrm{int}}-\frac{1}{2}=0 ;
$$


the spacetime energy of this state, as defined on the covering space, is then

$$
\mathcal{L}_{0}=J_{0}^{3}=\frac{k}{2} w+m=\frac{k w}{4}+\frac{1}{w}\left(\frac{1+4 s^{2}}{4 k}+h_{\text {int }}-\frac{1}{2}\right) .
$$

Before the orbifold, only $w \in \mathbb{Z}$ is allowed, and the threshold of the continuum

occurs for $w=1, s=0$, and $h_{\text {int }}=\frac{1}{2}$ (due to chiral GSO), at $\mathcal{L}_{0}=\frac{k^{2}+1}{4 k}$. The orbifold allows fractional spectral flow $w=\frac{p}{N}$, provided that one spectrally flows an amount $-p / N$ in $S U(2)$. This flows $h_{\text {int }}=\frac{1}{2}$ to $h_{\text {int }}=\frac{1}{2}+\frac{k}{4} w^{2}$ according to the $S U(2)$ version of (45); the spacetime energy of this state becomes

$$
\mathcal{L}_{0}=\frac{k w}{2}+\frac{1}{4 k w} .
$$

For $w=1 / N$, the energy is indeed reduced by an amount of order $N$.

\subsection{The $A d S_{3} / \mathbb{Z}_{N}$ Orbifold of the Superstring}

The orbifold of $A d S_{3}$ alone breaks supersymmetry in the superstring. The twist operators are the superparafermions $\hat{\Psi}_{j_{q}, j_{q}, j_{q}}^{S L(2)}$ of $S L(2, \mathbb{R})$. Their dimension is $k q / 2 N<$ $1 / 2$ and thus the NS sector twist operators describe tachyonic excitations of the orbifold point; the ground state is unstable. The twisted sector RR ground state operators take the form

$\left.e^{-\varphi / 2} \hat{\Psi}_{j_{q}, j_{q}, j_{q}}^{S L(2)} \exp \left[\epsilon_{1}\left(\frac{1}{2} \sqrt{\frac{2}{k}} \mathcal{X}+\frac{i}{2} \sqrt{\frac{\tilde{k}}{k}}\left(\frac{k}{\tilde{k}}-\frac{2 q}{N}\right) \mathcal{H}_{1}+\frac{i}{2} H_{2}\right)+\frac{i}{2} H_{3}+\epsilon_{4} \frac{i}{2}\left(H_{4}+H_{5}\right)\right)\right]$

where we have suppressed the right-moving free bosons. These are massless gauge fields coupling to the conserved RR charge of fractional D-branes (see section (5)). At the orbifold point, the vacuum is unstable, but the fractional D-branes couple to K-theoretic topological charges; the D-branes themselves are stable. As mentioned in the introduction, if the configuration is to decay to some superposition of states in the standard $A d S_{3} \times S^{3}$ string theory, the fate of these charges must be understood.

\section{D-branes}

Another class of excitations of the conical defect are fractional D-branes. D-branes at an orbifold singularity $\mathbb{R}^{n} / \Gamma$ are classified by the representations of $\Gamma$, which characterize the orbifold action on Chan-Paton structure [38, 39, 40, 41]. Branes in a given irreducible representation are pinned to the orbifold point; however, assembling irreps into the regular representation, a moduli space develops that allows them to be moved off the orbifold point - in the case of $\mathbb{Z}_{k}$, one needs $k$ fractional D-branes to put one brane on each leaf of the covering space away from the origin.

In flat spacetime, the tension of a $D p$-brane is $\mu_{p}=\left(g_{s} \ell_{s}^{p+1}\right)^{-1}$; however, in the presence of the nontrivial background ( $\mathbb{1 0}$ ), the energetics is modified due to 
binding energy with the background fundamental strings and NS fivebranes [27. For example, in the IIB theory, the left-moving energies of D-branes wrapping the $T^{4}$ are

$$
\mathcal{L}_{0}=\frac{1}{4 k} \sum_{i=1}^{4}\left(\frac{w_{i}^{D 3} \sqrt{v_{4}}}{r_{i}}+w_{D 1}^{i} \frac{r_{i}}{\sqrt{v}_{4}}\right)^{2} .
$$

Here $w_{i}^{D 3}=\frac{1}{6} \epsilon_{i j k l} w_{D 3}^{j k l}$ is the winding charge of D3-branes, and $w_{D 1}^{i}$ is the wrapping charge of D1-branes; $r_{i}$ are the radii of a rectangular $T^{4}$, and $v_{4}=r_{1} r_{2} r_{3} r_{4}$. We can then orbifold $A d S_{3} \times S^{3}$ by $\mathbb{Z}_{N}$, and passing to the IIA theory to turn odd branes into even ones, place fractional branes along the orbifold singularity, which is extended along one direction of the $S^{3}$; similar D-branes stretched across a great circle of $S^{3}$ have been considered recently in [42]. The size of the $S^{3}$ is $\sqrt{k}$ in string units, so (100) should be multiplied by $\sqrt{k}$. Similarly, in the IIB theory one has D-branes that lie along the one dimension of the orbifold singularity in $S^{3}$ and also wrap zero, two, or all four directions of $T^{4}$

$$
\mathcal{L}_{0}=\frac{1}{4 \sqrt{k}}\left(\frac{w_{D 1}}{\sqrt{v_{4}}}+w_{D 5} \sqrt{v_{4}}\right)^{2}+\frac{1}{4 \sqrt{k}} \sum_{i<j}\left({ }^{*} w_{i j}^{D 3} \frac{\sqrt{v_{4}}}{r_{i} r_{j}}+w_{D 3}^{i j} \frac{r_{i} r_{j}}{\sqrt{v}_{4}}\right)^{2} .
$$

Assembling fractional branes into regular representations does not result in a true moduli space for the brane in $\left(A d S_{3} \times S^{3}\right) / \mathbb{Z}_{N}$; due to the gravitational redshift of anti-de Sitter space, it costs energy to move an object away from the origin. However, the associated energy cost decreases from string scale for fractional branes to the scale set by the $A d S$ radius of curvature.

\section{Moduli Space}

In appropriately scaled variables, in the limit $k \rightarrow \infty$ the sigma model tends to flat spacetime. Consequently, the supersymmetric orbifold of the previous section degenerates to $\mathbb{R}^{1,1} \times\left(\mathbb{R}^{4} / \mathbb{Z}_{N}\right)$. String theory on the orbifold $\mathbb{R}^{4} / \mathbb{Z}_{N}$ has $4(N-1)$ moduli corresponding to the resolution of the orbifold singularity (c.f. 443, 44]). The resolution by blowing up inserts $N-1$ two-spheres. The moduli parametrize the hyperKähler blowup modes together with the B-flux through the two-spheres; in particular, the orbifold point corresponds to a set of collapsed two-spheres each threaded by a half unit of $B$-flux 43 .

From the construction of the twisted sector spectrum, we see that the $A d S_{3} \times$ $S^{3} / \mathbb{Z}_{N}$ orbifold also has a set of $4(N-1)$ massless modes corresponding to the operators (89). The orbifold effectively acts only on the coset theory $\frac{S L(2, \mathbb{R})}{U(1)} \times \frac{S U(2)}{U(1)}$, which is an $\mathcal{N}=(4,4)$ worldsheet superconformal field theory. The $\mathcal{N}=4$ algebra 455] is generated by the stress tensor $T(z)$, four supercurrents $G^{a \alpha}(z)$, and the $S U(2)$ R-symmetry current $J^{\alpha \beta}(z)$ (and corresponding antiholomorphic currents); the supercurrents additionally transform as a doublet under a global $S U(2)_{l}$ (the $a$ index). The $N-1$ moduli multiplets from the twisted sector transform as $(2,2)$ under this 
global $S U(2)_{l} \times S U(2)_{r}$ symmetry of the small $\mathcal{N}=(4,4)$ superconformal algebra, and are R-symmetry singlets. Since the $N-1$ quartets of massless fields all preserve $\mathcal{N}=4$ worldsheet supersymmetry, all these deformations are exactly marginal (the trace of the stress tensor lies in the same supermultiplet as the anomaly in the $S U(2)$ R-symmetry, which is not renormalized). Four additional massless multiplets arise from the untwisted sector and are universal, being obtained by worldsheet spectral flow [46] from the identity sector (see [47] for a recent review). These are the spin one-half representations of the $S U(2)$ R-symmetry current algebra, associated in spacetime to the supergravity multiplet. However, this last multiplet is not part of the moduli space of the orbifold - the expectation value of the zero momentum supergravity modes are not moduli on a noncompact space. All told, the $4(N-1)$ massless fields parametrize the moduli space $O(4, N-1) / O(4) \times O(N-1)$. It would be interesting to know whether there are any global identifications (other than the integer periodicity in the $B$ flux).

For $k \gg N \gg 1$, the orbifold looks locally very much like $\mathbb{R}^{1,1} \times\left(\mathbb{R}^{4} / \mathbb{Z}_{N}\right) \times T^{4}$, and an approximate description of the resolved orbifold singularity as an ALE space is appropriate (up to distances of order $\sqrt{k} l_{s}$ from the orbifold point). The metric on the ALE space is

$$
\begin{aligned}
d s^{2} & =V^{-1}(\vec{x})(d \tau+\vec{\omega} \cdot d \vec{x})^{2}+V(\vec{x}) d \vec{x} \cdot d \vec{x} \\
V(\vec{x}) & =\sum_{i=1}^{N}\left|\vec{x}-\vec{x}_{i}\right|^{-1},
\end{aligned}
$$

where $\vec{\nabla} V=\vec{\nabla} \times \vec{\omega}$. This should accurately describe the vicinity of the blowup when the deformation is not too far from the orbifold point in moduli space (which is $\vec{x}^{i}=0$ for all $i$ ). The $\vec{x}^{i}$ parametrize the three metric deformations of the collapsed spheres, and can roughly be thought of as the locations of the poles of the homology two-spheres of the resolved manifold (the fourth modulus being the $B$-flux through these homology two-spheres). These deformations might be thought of as certain kinds of breathing modes of the object in $A d S_{3}$. It is interesting that the object can have a large number of internal excitations, related to its mass.

\section{An aside on related models}

The orbifold action by the maximal discrete symmetry $\mathbb{Z}_{k}$ on the $(S L(2) / U(1)) \times$ $(S U(2) / U(1))$ sigma model was considered in a related context in [18]. The first factor $(S L(2) / U(1))$ is the Euclidean 2d black hole or 'cigar' sigma model of [49, 50], and also the theory of $S L(2, \mathbb{R})$ superparafermions; the second factor $(S U(2) / U(1))$ is the the $X^{k}$ Landau-Ginsburg theory [51, 52], or equivalently the $S U(2)$ superparafermion theory.] The orbifold $[(S L(2) / U(1)) \times(S U(2) / U(1))] / \mathbb{Z}_{k}$ was shown in [48, 53, 54] to give a CFT with a target space which is asymptotically $S^{3} \times \mathbb{R}$,

\footnotetext{
${ }^{9}$ The monomial $X^{q}$ of the Landau-Ginsburg field is the superparafermion $\hat{\Psi}_{j_{q}, j_{q}, j_{q}}^{S U(2)}$
} 
corresponding to the throat geometry of $k$ fivebranes. It was argued in these works that the target spacetime is algebraically described by the deformed $\mathbb{Z}_{k}$ singularity

$$
X^{k}+Y^{2}+Z^{2}=\mu \text {. }
$$

What is the relation among these various theories? The operators/states of the $S U(2)$ WZW model can be decomposed into a parafermion part and a free field part arising from the bosonization of $J^{3}$ :

$$
\Phi_{j m \bar{m}}^{\mathrm{SU}(2)}=\hat{\Psi}_{j m \bar{m}}^{S U(2)} \exp \left[i \sqrt{\frac{2}{k}}(n \mathcal{Y}+\bar{n} \overline{\mathcal{Y}})\right]
$$

with $m=n, \bar{m}=\bar{n}$, and $m, \bar{m}=-j, \ldots, j$. On the other hand, the tensor product theory $(S U(2) / U(1))_{\mathrm{pf}} \times U(1)_{\text {circ }}$ consists of the operators on the RHS of (104), with $m, \bar{m}$ independent of $n, \bar{n}$, and $(m-\bar{m}) \in k \mathbb{Z}$ (and similarly for $n-\bar{n}$ ). The parafermion operators respect a $\mathbb{Z}_{k}^{\mathrm{pf}} \times \tilde{\mathbb{Z}}_{k}^{\mathrm{pf}}$ symmetry, under which the operators carry the quantum numbers $\left(l, l^{\prime}\right)=(m+\bar{m}, m-\bar{m}) \bmod k$ [55]. Similarly considering a $\mathbb{Z}_{k}^{\text {circ }} \times \tilde{\mathbb{Z}}_{k}^{\text {circ }}$ subgroup of the $U(1) \times U(1)$ symmetry of the free boson, the $S U(2)$ WZW theory is the orbifold of the parafermion times $U(1)$ theory by the diagonal vectorlike $\mathbb{Z}_{k}^{\prime}=\left(\mathbb{Z}_{k}^{\mathrm{pf}} \times \mathbb{Z}_{k}^{\text {circ }}\right)_{\text {diag }}$

$$
S U(2)=\left[\left(\frac{S U(2)}{U(1)}\right)_{\mathrm{pf}} \times U(1)_{\operatorname{circ}}\right] / \mathbb{Z}_{k}^{\prime}
$$

under which the states (104) carry $\mathbb{Z}_{k}^{\prime}$ charge $(m+\bar{m}-n-\bar{n})$. The orbifold by $\mathbb{Z}_{k}^{\prime}$ sets $m=n, \bar{m}=\bar{n}$; the twisted sectors relax the condition $m-\bar{m} \in k \mathbb{Z}$ to $m-\bar{m} \in \mathbb{Z}$. The relation between the $S L(2, \mathbb{R})$ WZW model and the $\frac{S L(2, \mathbb{R})}{U(1)} \times \mathbb{R}$ cigar plus time background is not as straightforward; the parafermion states have spin $j$ taking continuous values $0 \leq j<\frac{k-1}{2}$. Nevertheless, the $\mathbb{Z}_{k}$ symmetry of (55) (for $k=N$ ) acts on the embedded parafermion model in precisely the same way as in 48, 53, 54].

There are thus two ways of getting at the orbifold theory we are discussing in this paper. Starting with the target

$$
(S L(2))_{\mathrm{WZW}} \times\left(\frac{S U(2)}{U(1)}\right)_{\mathrm{pf}} \times U(1)_{\mathrm{circ}} \times T^{4},
$$

one may construct $A d S_{3} \times S^{3}$ by orbifolding by $\mathbb{Z}_{k}^{\prime}$ and then performing the orbifold by the $\mathbb{Z}_{k}$ of (55), which acts on the embedded $S U(2)$ and $S L(2)$ parafermions of the respective WZW models. Alternatively, one can first orbifold by this latter $\mathbb{Z}_{k}$, to make a variant of the CHS model as in [48, 53, 54, and then quotient by the first symmetry $\mathbb{Z}_{k}^{\prime}$.

There are correspondingly a couple of ways to view the deformation along the moduli space of the orbifold singularity. One is (102), which gives an approximate description of the geometry near the orbifold singularity for not too large a blowup. 
On the other hand, for $k=N \gg 1$, the description of [48, 53, 54] in terms of Landau-Ginsburg models is perhaps more appropriate, and the geometry near the object is stringy.

The division of the target space into superparafermion and $U(1)$, as in (106), is the starting point for a more general orbifold using the construction of [33]. There, spacetime CFT vacua preserving at least eight supersymmetries are built using $c=15$ worldsheet superconformal field theories of the form

$$
A d S_{3} \times U(1)_{k} \times\left(\frac{\mathcal{M}}{U(1)}\right),
$$

where $\mathcal{M} / U(1)$ is $\mathcal{N}=2$ supersymmetric on the worldsheet, and the subscript $k$ is the 'level' of the $U(1)$ supercurrent algebra (the radius squared of the target space circle in units of the self-dual radius). In this paper we have concentrated on perhaps the simplest example $\mathcal{M} / U(1)=S U(2) / U(1) \times T^{4}$, but clearly one may generalize. $\mathrm{A} \mathbb{Z}_{N}$ orbifold preserving half the supersymmetry may be constructed along the lines of sections 3 and 4 , provided $N$ divides $k$.

\section{The Spacetime CFT}

There is an intimate relation between the asymptotic $A d S$ geometry of the string background and the conformal invariance of its dual CFT description [21, 56, 22, 8]. In the orbifold constuction described above, the quotient space description of the theory inherits a subalgebra of the $\mathcal{N}=4$ superconformal algebra on the covering space which is itself a twisted $\mathcal{N}=4$ superconformal algebra. The central charge of the quotient space superVirasoro algebra $c=N \tilde{c}$, where $\tilde{c}$ is the central charge that appears in the covering space description, is consistent with what is expected in gravity on asymptotically $A d S$ spacetimes. A factor of $1 / N$ appears in $\tilde{c}$ which is due to the fact that gravity as described on the covering space has a Planck scale that is $N$ times larger than that on the quotient space. The orbifold geometry can be seen to be the fractional spectral flow in the spacetime superconformal field theory of a Ramond vacuum state which carries $1 / N$ of the maximal allowed $\mathcal{T}_{0}^{3}$ charge of the Ramond ground states. Note that in this section, when we discuss spectral flow, we are referring to the flow of states in the spacetime superconformal field theory.

\subsection{The Spacetime Superconformal Algebra}

The spacetime conformal field theory of the onebrane-fivebrane system is a representation of the $\mathcal{N}=4$ superVirasoro algebra [45] This spacetime algebra on the

\footnotetext{
${ }^{10} \mathrm{At}$ the particular point in the moduli space realized by the perturbative worldsheet description, the spacetime CFT is singular; the representation theoretic aspects of this situation are not well understood. We thank D. Kutasov for emphasizing this point.
} 
covering space $A d S_{3} \times S^{3} \times T^{4}$ is as follows

$$
\begin{aligned}
{\left[\tilde{\mathcal{L}}_{n}, \tilde{\mathcal{L}}_{m}\right] } & =(n-m) \tilde{\mathcal{L}}_{n+m}+\frac{\tilde{c}}{12} n^{3} \delta_{n+m} \\
{\left[\tilde{\mathcal{T}}_{n}^{i}, \tilde{\mathcal{T}}_{m}^{j}\right] } & =i \epsilon^{i j k} \tilde{\mathcal{T}}_{n+m}^{k}+\frac{\tilde{c}}{12} n \delta^{i j} \delta_{n+m} \\
{\left[\tilde{\mathcal{L}}_{n}, \tilde{\mathcal{T}}_{m}^{i}\right] } & =-m \tilde{\mathcal{T}}_{n+m}^{i} \\
\left\{\tilde{\mathcal{G}}_{r}^{* \alpha}, \tilde{\mathcal{G}}_{s}^{\beta}\right\} & =2 \delta_{\alpha \beta} \tilde{\mathcal{L}}_{r+s}-2(r-s) \sigma_{\alpha \beta}^{i} \tilde{\mathcal{T}}_{r+s}^{i}+\frac{\tilde{c}}{12} 4 r^{2} \delta_{\alpha \beta} \delta_{r+s} \\
{\left[\tilde{\mathcal{T}}_{n}^{i}, \tilde{\mathcal{G}}_{r}^{\alpha}\right] } & =\frac{1}{2} \sigma_{\beta \alpha}^{i} \tilde{\mathcal{G}}_{n+r}^{\beta} \\
{\left[\tilde{\mathcal{L}}_{n}, \tilde{\mathcal{G}}_{r}^{\alpha}\right] } & =-\left(r-\frac{1}{2} n\right) \tilde{\mathcal{G}}_{n+r}^{\alpha} .
\end{aligned}
$$

All other (anti-) commutators either vanish or are implied by the relation $\left(\tilde{\mathcal{G}}_{r}^{\alpha}\right)^{\dagger}=$ $\tilde{\mathcal{G}}_{-r}^{* \alpha}$. The moding of the supercharges is that of the NS sector associated with global $A d S_{3} \times S^{3}$. That is $r \in \mathbb{Z}+1 / 2$ for $\tilde{\mathcal{G}}_{r}^{\alpha}$ and $n \in \mathbb{Z}$ for $\tilde{\mathcal{T}}_{n}^{ \pm}$. The central terms appearing here differ slightly from standard CFT conventions due to the $A d S_{3}$ convention that the $S L(2, \mathbb{R})$ invariant vacuum state has energy $\tilde{\mathcal{L}}_{0}=-\frac{\tilde{c}}{24}$ rather than zero. Expressions for the generators of this algebra in terms of the fields of the sigma model on (Euclidean) $A d S_{3} \times S^{3}$ have been given in 8, 57, 58]. The rule of thumb is that any (anti)holomorphic algebra on the worldsheet is related to a corresponding algebra in spacetime; roughly, long strings near the boundary of $A d S_{3}$ have the spacetime algebra pulled back onto the worldsheet.

The $\mathbb{Z}_{N}$ orbifold of spacetime projects the generators (108) onto the subalgebra which commute with the $\mathbb{Z}_{N}$ action generated by

$$
\exp \left[\frac{2 \pi i}{N}\left(\tilde{\mathcal{L}}_{0}-\tilde{\overline{\mathcal{L}}}_{0}-\tilde{\mathcal{T}}_{0}^{3}+\tilde{\mathcal{\mathcal { T }}}_{0}^{3}\right)\right]
$$

For example, of the Virasoro and R-current generators $\tilde{\mathcal{L}}_{n}$ and $\tilde{\mathcal{T}}_{n}^{3}$, one keeps only those with $n \in N \mathbb{Z}$; similarly $\tilde{\mathcal{G}}_{r}^{ \pm}$survive for $r \pm \frac{1}{2} \in N \mathbb{Z}$ (so that $\tilde{\mathcal{G}}_{\mp 1 / 2}^{ \pm}$are the surviving supersymmetries), and we also keep $\tilde{\mathcal{T}}_{n}^{ \pm}$for $n \pm 1 \in N \mathbb{Z}$. Here we have defined $\tilde{\mathcal{G}}_{r}^{+} \equiv \tilde{\mathcal{G}}_{r}^{1}$ and $\tilde{\mathcal{G}}_{r}^{-} \equiv \tilde{\mathcal{G}}_{r}^{2}$ to reflect the $\tilde{\mathcal{T}}_{0}^{3}$ eigenvalue. We similarly define $\tilde{\mathcal{G}}_{r}^{*+} \equiv \tilde{\mathcal{G}}_{r}^{* 2}$ and $\tilde{\mathcal{G}}_{r}^{*-} \equiv \tilde{\mathcal{G}}_{r}^{* 1}$. Note that the effect of the orbifold on the spacetime CFT is not simply to project onto the states that are invariant under (109); this would allow multiparticle states invariant under (109) built out of particles that were not individually invariant under (109) (for instance, operators in the Virasoro enveloping algebra built out of products of Virasoro raising operators whose total level is a multiple of $N$ but whose component raising operators have levels that are not multiples of $N$ ). Such states are not present in the orbifold.

In the twisted sector of the orbifold, no new (anti)holomorphic currents appear on the worldsheet, and therefore one expects that no new symmetries of spacetime will appear. The $\mathbb{Z}_{N}$ projected superVirasoro algebra is then the full set of symmetries of the spacetime. We may define the following spacetime generators associated with a twisted superconformal symmetry on the quotient space

$$
\mathcal{L}_{n}=\frac{1}{N} \tilde{\mathcal{L}}_{n N}
$$




$$
\begin{aligned}
& \mathcal{T}_{n}^{3}=\tilde{\mathcal{T}}_{n N}^{3} \\
& \mathcal{T}_{n \mp \frac{1}{N}}^{ \pm}=\tilde{\mathcal{T}}_{n N \mp 1}^{ \pm} \\
& \mathcal{G}_{n \mp \frac{1}{2 N}}^{ \pm}=\frac{1}{\sqrt{N}} \tilde{\mathcal{G}}_{n N \mp \frac{1}{2}}^{ \pm} .
\end{aligned}
$$

Taking $c=N \tilde{c}$, these generators satisfy the following algebra

$$
\begin{aligned}
{\left[\mathcal{L}_{n}, \mathcal{L}_{m}\right] } & =(n-m) \mathcal{L}_{n+m}+\frac{c}{12} n^{3} \delta_{n+m} \\
{\left[\mathcal{T}_{n}^{3}, \mathcal{T}_{m}^{3}\right] } & =\frac{c}{12} n \delta_{n+m} \\
{\left[\mathcal{T}_{n}^{3}, \mathcal{T}_{m \mp \frac{1}{N}}^{ \pm}\right] } & = \pm \mathcal{T}_{n+m \mp \frac{1}{N}}^{ \pm} \\
{\left[\mathcal{T}_{n-\frac{1}{N}}^{+}, \mathcal{T}_{m+\frac{1}{N}}^{-}\right] } & =2 \mathcal{T}_{m+n}^{3}+2 \frac{c}{12}\left(n-\frac{1}{N}\right) \delta_{n+m} \\
{\left[\mathcal{L}_{n}, \mathcal{T}_{m}^{3}\right] } & =-m \mathcal{T}_{n+m}^{3} \\
{\left[\mathcal{L}_{n}, \mathcal{T}_{m \mp \frac{1}{N}}^{ \pm}\right] } & =-\left(m \mp \frac{1}{N}\right) \mathcal{T}_{n+m \mp \frac{1}{N}}^{ \pm} \\
\left\{\mathcal{G}_{n \mp \frac{1}{2 N}}^{* \pm}, \mathcal{G}_{m \mp \frac{1}{2 N}}^{ \pm}\right\} & =-2(n-m) \mathcal{T}_{n+m \mp \frac{1}{N}}^{ \pm} \\
\left\{\mathcal{G}_{n \mp \frac{1}{2 N}}^{* \pm}, \mathcal{G}_{m \pm \frac{1}{2 N}}^{\mp}\right\} & =2 \mathcal{L}_{n+m} \pm 2\left(n-m \mp \frac{1}{N}\right) \mathcal{T}_{n+m}^{3}+\frac{c}{12} 4\left(n \mp \frac{1}{2 N}\right)^{2} \delta_{n+m} \\
{\left[\mathcal{T}_{n}^{3}, \mathcal{G}_{m \mp \frac{1}{2 N}}^{ \pm}\right] } & = \pm \mathcal{G}_{n+m \mp \frac{1}{2 N}}^{ \pm} \\
{\left[\mathcal{T}_{n \mp \frac{1}{N}}^{ \pm}, \mathcal{G}_{m \pm \frac{1}{2 N}}^{\mp}\right] } & =\mathcal{G}_{n+m \mp \frac{1}{2 N}}^{ \pm} \\
{\left[\mathcal{L}_{n}, \mathcal{G}_{m \mp \frac{1}{2 N}}^{ \pm}\right.} & =-\left(m \mp \frac{1}{2 N}-\frac{1}{2} n\right) \mathcal{G}_{n+m \mp \frac{1}{2 N}}^{ \pm}
\end{aligned}
$$

Thus we can define generators which satisfy a form of the $\mathcal{N}=4$ superVirasoro algebra for $c=N \tilde{c}$, spectrally flowed [46] by $-1 / N$ units from Ramond boundary conditions.

A number of issues arise from the form of the above quotient space superconformal algebra. One is the question of the spacetime $\mathcal{L}_{0}$ and $\mathcal{T}_{0}^{3}$ charges of the orbifold geometries themselves, in a suitably chosen asymptotic frame, in the $S U(1,1 \mid 2) \times S U(1,1 \mid 2)$ supergravity that (naively) arises from a Kaluza-Klein reduction on $S^{3}$. Related to this is the issue of how the above moding of the supercharges is related to the $S U(2)$ gauge field of the extended supergravity and how spectral flow enters the picture. Another question is the appearance of the factor $c=N \tilde{c}$ in the superalgebra and its interpretation in the boundary theories on the covering and quotient spacetimes. We will answer these questions in the next two sections but will provide here a simplified description of the relationship between the covering and quotient space algebras in the case of an bosonic orbifold of $A d S_{3}$ without a corresponding twisting of the $S^{3}$. That is we consider a metric on the covering space of the form

$$
d \tilde{s}^{2} / k=-\left(1+\tilde{r}^{2}\right) d \tilde{t}^{2}+\left(1+\tilde{r}^{2}\right)^{-1} d \tilde{r}^{2}+\tilde{r}^{2} d \tilde{\phi}^{2} .
$$


Under the orbifold identification $\tilde{\phi} \sim \tilde{\phi}+\frac{2 \pi}{N}$ with the following coordinate transformation

$$
t=N \tilde{t}, \quad r=\tilde{r} / N, \quad \phi=N \tilde{\phi},
$$

we find the following metric on the quotient spacetime

$$
d s^{2} / k=-\left(N^{-2}+r^{2}\right) d t^{2}+\left(N^{-2}+r^{2}\right)^{-1} d r^{2}+r^{2} d \phi^{2} .
$$

More generally this coordinate transformation defines a map between metrics which have angular periodicity $2 \pi / N$ and are asymptotically $A d S$ on the covering space and metrics which have angular periodicity $2 \pi$ and are asymptotically $A d S$ on the quotient space. To see that the above form of the relation between the spacetime generators as defined on the covering space and those defined on the orbifold is the correct one, we examine the transformation properties of the worldsheet form of the superconformal generators. The generators of the asymptotic symmetries of asymptotically $A d S_{3}$ spacetimes can be derived from the sigma model action via a Noether procedure. The generators of the bosonic Euclidean $S L(2, \mathbb{R}) \times S U(2)$ WZW model will be discussed briefly here. The Euclidean version of the $S L(2, \mathbb{R})$ theory is the $\mathrm{H}_{3}^{+}=S L(2, \mathbb{C}) / S U(2)$ coset model. Diffeomorphisms will, when acting on physical vertex operators, lead to states that are the same element in the worldsheet BRST cohomology. This is not the case for the asymptotic symmetries of $A d S_{3}$ since these are not globally well defined diffeomorphisms. In particular, the set of geometries given by the action of the symmetries on global $A d S_{3}$ are physically distinguishable and span the space of all asymptotically $A d S_{3}$ spacetimes. Holomorphic contour integral representations of the operators may be derived for $\mathcal{L}_{n}$ and $\mathcal{T}_{n}^{a}$. We express these generators in the $(\tau=i t, r, \phi)$ coordinates in the limit $r \rightarrow \infty$. Taking $w=\tau+i \phi$ we find

$$
\begin{aligned}
\mathcal{L}_{n} & =\frac{k}{2 \pi i} \oint d z e^{-n \bar{w}}\left(r^{2} \partial w+n \frac{\partial r}{r}\right) \\
\mathcal{T}_{n}^{a} & =\frac{1}{2 \pi i} \oint d z e^{-n \bar{w}} K^{a} .
\end{aligned}
$$

Where $K^{a}$ is the holomorphic $S U(2)$ current. Using the transformation between covering space and quotient space variables given above $(r=\tilde{r} / N$ and $w=N \tilde{w})$ it may be seen that $\tilde{\mathcal{L}}_{N n} / N=\mathcal{L}_{n}$ and $\tilde{\mathcal{T}}_{N n}^{a}=\mathcal{T}_{n}^{a}$, in agreement with the expressions given above for the superconformal generators. For instance, for the $S L(2, \mathbb{R})$ invariant NS ground state, $\tilde{\mathcal{L}}_{0}=-\tilde{c} / 24$ and $\mathcal{L}_{0}=-c /\left(24 N^{2}\right)=\tilde{\mathcal{L}}_{0} / N$. Here we have determined $\tilde{\mathcal{L}}_{0}$ and $\mathcal{L}_{0}$ from the form of the metric. In general the following metric, in quotient space variables, describes the conical spacetimes as well as the BTZ black holes

$$
\begin{aligned}
& d s^{2}=-N_{t}^{2} d t^{2}+N_{t}^{-2} d r^{2}+r^{2}\left(d \phi-N_{\phi} d t\right)^{2} \\
& N_{t}^{2}=\frac{r^{2}}{\ell^{2}}-8 \ell_{p} M+\frac{16 \ell_{p}^{2} J^{2}}{r^{2}}
\end{aligned}
$$




$$
N_{\phi}=\frac{4 \ell_{p} J}{r^{2}}
$$

Geometries with $M>0$ are black holes, while those with $M<0$ are conical defects. Using $c=3 \ell / 2 \ell_{p}$ we have $\ell M=\mathcal{L}_{0}+\overline{\mathcal{L}}_{0}$ and $J=\mathcal{L}_{0}-\overline{\mathcal{L}}_{0}$. As will be seen below, this relationship will be modified when we consider gauge fields arising from the $S^{3}$ fibration.

\subsection{The Spacetime Charges and Spectral Flow}

In the case of the supersymmetric orbifold considered above, the charges measured by an asymptotic observer are modified as described in [59, 17, 16]. It is first necessary to specify the relationship between the covering and quotient space coordinates. We begin with the metric on $A d S_{3} \times S^{3}$ expressed in covering space coordinates. Taking the length scales to be identical

$$
\begin{aligned}
d \tilde{s}^{2} / k= & -\left(1+\tilde{r}^{2}\right) d \tilde{t}^{2}+\left(1+\tilde{r}^{2}\right)^{-1} d \tilde{r}^{2}+\tilde{r}^{2} d \tilde{\phi}^{2} \\
& +\cos ^{2} \tilde{\chi} d \tilde{\theta}^{2}+d \tilde{\chi}^{2}+\sin ^{2} \tilde{\chi} d \tilde{\psi}^{2} .
\end{aligned}
$$

The $S^{3}$ coordinate $\tilde{\psi}$ was given by $\phi_{\mathrm{Su}(2)}$ above (see equation (54)) and the $A d S$ metric takes the form (10) when $\tilde{r}=\sinh \tilde{\rho}$. The orbifold is given by the identification $(\tilde{\phi}, \tilde{\psi}) \sim\left(\tilde{\phi}+\frac{2 \pi}{N}, \tilde{\psi}-\frac{2 \pi}{N}\right)$. We may define new coordinates on the quotient spacetime

$$
\begin{aligned}
& t=N \tilde{t}, \quad r=\tilde{r} / N, \quad \phi=N \tilde{\phi}, \\
& \theta=\tilde{\theta}, \quad \chi=\tilde{\chi}, \quad \psi=\tilde{\psi}+\tilde{\phi},
\end{aligned}
$$

which may be seen to satisfy $(\phi, \psi) \sim(\phi+2 \pi, \psi) \sim(\phi, \psi+2 \pi)$. This leads to the quotient space metric

$$
\begin{aligned}
d s^{2} / k= & -\left(N^{-2}+r^{2}\right) d t^{2}+\left(N^{-2}+r^{2}\right)^{-1} d r^{2}+r^{2} d \phi^{2} \\
& +\cos ^{2} \chi d \theta^{2}+d \chi^{2}+\sin ^{2} \chi\left(d \psi-N^{-1} d \phi\right)^{2} .
\end{aligned}
$$

The $B$ field is of course similarly modified. Note that this choice of coordinates establishes the charges of the orbifold spacetimes with respect to the associated asymptotic frame. It will be shown that this particular choice places the orbifold in the Ramond sector of boundary CFT. Any other choice that is related by a globally well-defined coordinate transformation is equivalent but will lead to a different asymptotic frame with different global charges. The Kaluza-Klein reduction of the metric and $B$ fields give rise to a pair of gauge fields in $S U(1,1 \mid 2) \times S U(1,1 \mid 2)$ supergravity. Following [16], these are given by

$$
A=\bar{A}=-N^{-1} \sigma^{3} d \phi
$$


Furthermore the global charges are modified by the presence of the gauge fields. Let us define the global charges $Q^{a}$

$$
Q^{a}=\frac{1}{2 \pi} \oint \frac{1}{2} \operatorname{tr}\left[\sigma^{a} A\right]
$$

And similarly for $\bar{Q}^{a}$. In terms of these charge and the charges $M$ and $J$, which may be derived similarly by expressing the Einstein-Hilbert action in terms of gauge fields for a $S L(2, \mathbb{R}) \times S L(2, \mathbb{R})$ Chern-Simons theory, the global charges are given by

$$
\begin{aligned}
\mathcal{T}_{0}^{3} & =\frac{c}{12} Q^{3} \\
\overline{\mathcal{T}}_{0}^{3} & =\frac{c}{12} \bar{Q}^{3} \\
\mathcal{L}_{0} & =\frac{1}{2}(\ell M+J)+\frac{c}{24} Q^{a} Q^{a} \\
\overline{\mathcal{L}}_{0} & =\frac{1}{2}(\ell M-J)+\frac{c}{24} \bar{Q}^{a} \bar{Q}^{a} .
\end{aligned}
$$

These relations lead to the charges $\mathcal{T}_{0}^{3}=\overline{\mathcal{T}}_{0}^{3}=-\frac{c}{12} N^{-1}$ and $\mathcal{L}_{0}=\overline{\mathcal{L}}_{0}=0$. The orbifold thus lies in the Ramond sector of the spacetime CFT when charges are defined with respect to the choice of quotient space frame given by (118). Note that had we chosen the asymptotic frame $\psi=\tilde{\psi}+(N-1) \tilde{\phi}$ we would have found $A=\bar{A}=\left(1-N^{-1}\right) \sigma^{3} d \phi$. This choice identifies the orbifold spacetimes as being chiral primary states in the NS sector of the spacetime CFT, with charges $\mathcal{T}_{0}^{3}=$ $\mathcal{L}_{0}+\frac{c}{24}=\frac{c}{12}\left(1-N^{-1}\right)$. 10 How does this analysis comport with the conclusion, based on the relationship between the superconformal generators (110), that $\mathcal{L}_{0}=$ $-c /\left(24 N^{2}\right)=\tilde{\mathcal{L}}_{0} / N$ and $\mathcal{T}_{0}^{3}=\tilde{\mathcal{T}}_{0}^{3}=0$ ? These latter values for the charges follow from the fact that the covering space - global $A d S_{3}$ - has charges $\tilde{\mathcal{L}}_{0}=-\tilde{c} / 24$ and $\tilde{\mathcal{T}}_{0}^{3}=0$. The answer is that the superalgebra (111) corresponds to gauging away the $A$ and $\bar{A}$ fields for each choice of $N$. This is just the operation of spectral flow in the boundary CFT which produces the moding of the supercharges seen in (111). Spectral flow induces the shifts

$$
\begin{aligned}
\mathcal{L}_{0}(\eta) & =\mathcal{L}_{0}-\mathcal{T}_{0}^{3} \eta+\frac{c}{24} \eta^{2} \\
\mathcal{T}_{0}^{3}(\eta) & =\mathcal{T}_{0}^{3}-\frac{c}{12} \eta .
\end{aligned}
$$

The Ramond vacuum state which is the $\eta=1$ spectral flow of the NS groundstate thus has $\mathcal{L}_{0}=0$ and $\mathcal{T}_{0}^{3}=-c / 12$. Starting with a state with charges $\mathcal{L}_{0}=$

\footnotetext{
${ }^{11}$ The identification of these coordinate choices with the R and NS sectors may be seen by expressing a $2 \pi$ rotation in $\psi$ in the respective quotient space frames in terms of group actions on the covering space. The action of the respective group elements on the supercharges (71) will produce -1 for the NS sector (as it does for global $A d S_{3}$ ) and +1 for the $\mathrm{R}$ sector.
} 
0 and $\mathcal{T}_{0}^{3}=-c /(12 N)$, which are the charges with respect to the gauge choice corresponding to (118), a fractional spectral flow by $-1 / N$ leads to

$$
\mathcal{L}_{0}=-\frac{c}{24 N^{2}} \quad \text { and } \quad \mathcal{T}_{0}^{3}=0
$$

which are the charges with respect to the superalgbra (111). Note that it is more consistent to express the charges of the orbifolds with respect to a particular asymptotic frame which places them in the same spectral flow sector. The charges (124) correspond to a different gauge choice for each orbifold and thus to a different choice of (in general only locally asymptotically $A d S_{3}$ ) quotient space coordinate system for each. However, keeping this in mind, we may choose to express the charges of the orbifolds in terms of (111). Figure 2 shows a plot of the $\mathcal{L}_{0}$ and $\mathcal{T}_{0}^{3}$ charges of the superalgebra (111) for the $N=2$ orbifold. The other orbifolds may be seen to lie along the line given by the spectral flow of the $\left(\mathcal{L}_{0}=\mathcal{T}_{0}^{3}-\frac{c}{24}\right)$ chiral primary states. Also shown in the plot is the unitarity bound given by $\mathcal{L}_{0}=(6 / c)\left(\mathcal{T}_{0}^{3}\right)^{2}-\frac{c}{24}$ that applies to all states in all of the spectral flow sectors of the superconformal algebra.

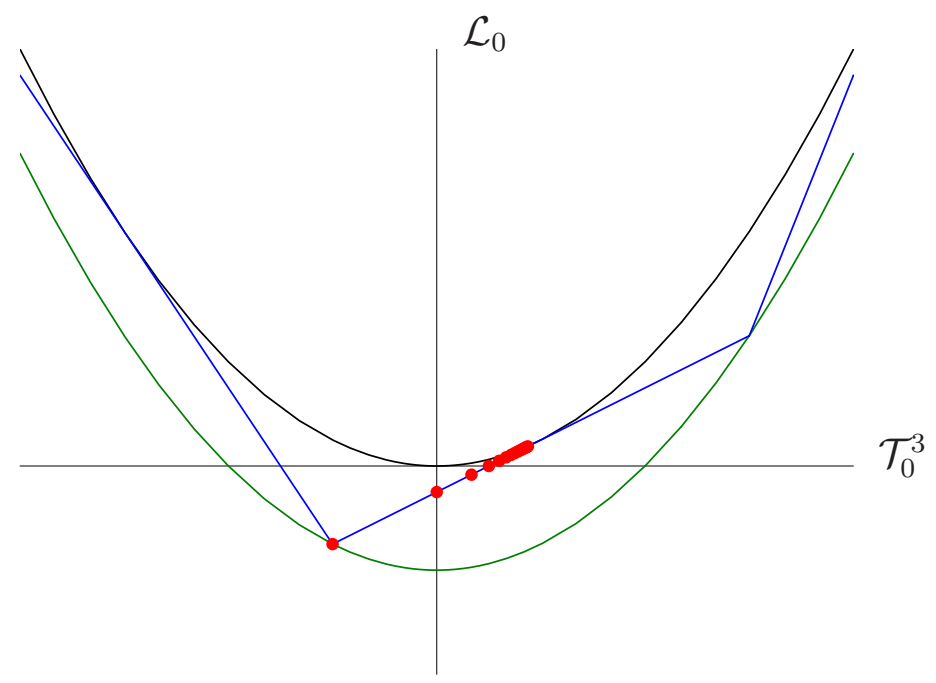

Figure 2: Charges of the spectral flow of the chiral primary states (the red dots) associated with the orbifold spacetimes for the sector corresponding to the $N=2$ orbifold. The plot shows the unitarity bound (the circumscribed green parabola) on all of the states of the $\mathcal{N}=4$ superconformal algebra as well as the more restrictive bound (the blue polygon) that applies to the sector of the $N=2$ orbifold. The plot also shows the lower bound (the inscribed black parabola) for the emergence of BTZ black holes. 


\subsection{The Spacetime Central Charge}

The possibilty for confusion exists over the factor of $N$ in the relationship ( $c=$ $N \tilde{c})$ between the central charge in the covering space and that in the quotient spacetime.[2] The issue is seen to appear in the description of low energy gravity on the respective spacetimes. Ignoring surface terms, consider the Einstein-Hilbert action on the quotient spacetime

$$
S_{\mathrm{EH}}=\frac{1}{16 \pi \ell_{p}} \int d t d r \int_{0}^{2 \pi} d \phi \sqrt{-g} R[g]
$$

We are considering metrics which are asymptotically $A d S_{3}$ on the quotient space with periodicity $2 \pi$ in the angular coordinate $\phi$. We now write this action in the covering space coordinates given by the transformation (113). Since the metric has periodicity $2 \pi / N$ in the angular coordinate $\tilde{\phi}$, we may integrate over the entire covering space while dividing by $N$ and replacing the quotient space $\ell_{p}$ by the corresponding scale on the covering space $\tilde{\ell}_{p}=N \ell_{p}$

$$
S_{\mathrm{EH}}=\frac{1}{16 \pi \tilde{\ell_{p}}} \int d \tilde{t} d \tilde{r} \int_{0}^{2 \pi} d \tilde{\phi} \sqrt{-\tilde{g}} R[\tilde{g}]
$$

The asymptotic Virasoro algebra on $A d S_{3}$ may be derived from either of these forms of the action with the understanding that there is a restriction on the space of metrics defined on the covering space to those that have $2 \pi / N$ periodicity in the angular coordinate $\tilde{\phi}$. As described above, this will project out those generators of the algebra which do not respect this periodicity. The central charge of the Virasoro algebra on the covering space will be $\tilde{c}=3 \ell / 2 \tilde{\ell}_{p}=c / N$. Thus the introduction of the factor of $N$ into the central charge of the algebra (111) corresponds to a return to the central charge of the quotient spacetime. Another way to see the emergence of the factor of $N$ in the central charge on the covering space is to note that in the formula $c=6 k p$ the integer $p$ represents the number of fundamental strings which produce the background. If there are $\tilde{p}$ of these on the covering space then there will be $p=N \tilde{p}$ on the orbifold since each of the $\tilde{p}$ strings will wind $N$ times around the quotient spacetime. The same factor of $N$ may be derived using the transformation (113) from the worldsheet vertex operators associated with the central charge of the Virasoro algebra derived in $8,57,58$.

BTZ black holes may be constructed by dumping energy into the system. These have an entropy

$$
S=\frac{A}{4 \ell_{p}}=2 \pi\left(\frac{c}{6} \mathcal{L}_{0}-\left(\mathcal{T}_{0}^{3}\right)^{2}\right)^{1 / 2}+2 \pi\left(\frac{c}{6} \overline{\mathcal{L}}_{0}-\left(\overline{\mathcal{T}}_{0}^{3}\right)^{2}\right)^{1 / 2}
$$

\footnotetext{
${ }^{12}$ In particular, it is possible to draw erroneous conclusions about the aymptotic growth of the density of states in the spacetime CFT and consequences for black hole entropy. Evidence for the existence of such a pitfall is given by statements in the original version of this paper.
} 
which is invariant under spectral flow. The BTZ black hole threshold is indicated in Figure 2; it touches the line of BPS states midway between its extremes. The orbifold geometries thus approach the black hole threshold from below at large $N$.

For the non-supersymmetric orbifold, we may approach the black hole threshold arbitrarily closely, since there is no bound on $N$. For the supersymmetric orbifold we get closest to the BTZ threshold by taking $N=k$. The energy below the threshold at this point is of order $p k / N^{2}=p / k=g_{6}^{-2}$ in units of the AdS radius. One is still a macroscopic distance in configuration space from the BTZ black hole threshold.

Even though we cannot approach the density of states of the BTZ black hole in a regime where low-energy supergravity is reliable, the $N=k$ orbifold does have a large number of degrees of freedom concentrated in the vicinity of the orbifold singularity, and it will be interesting to see to what extent the properties of the orbifold parallel those of the black hole that arises at higher mass.

\section{Summary}

We have shown that the $S L(2)$ and $S U(2)$ (super)parafermion theories [30, 55, 34] provide a natural formulation of the $\mathbb{Z}_{N}$ orbifold of string theory on $A d S_{3} \times S^{3}$ (times $T^{4}$ or $K 3$ ). The spacetime so constructed is a kind of embedding of an ALE singularity into $A d S_{3} \times S^{3}$. Twisted sector vertex operators are built out of the

parafermion highest weight states, and the spectral flow operation used [31, 33] to fill out the single-string spectrum extends to a fractional spectral flow generating radially oscillating strings that close only up to the orbifold identification. The $N-1$ twisted sectors provide $4(N-1)$ new moduli of the worldsheet CFT, analogous to the hyperKähler blowup modes of an ALE singularity. These may have an interpretation as describing a $4(N-1)$ parameter family of states in the spacetime CFT.

We also explored the symmetry algebra of the spacetime CFT and the associated black hole entropy formula. The orbifold spacetime is a representation of a fractionally moded $\mathcal{N}=(4,4)$ superconformal algebra; the fractional moding can be obtained by $1 / N$ spectral flow from Ramond boundary conditions. Flowing back to Ramond boundary conditions, the collection of orbifolds of various $N$ describe a discrete set of BPS states in the spacetime CFT, corresponding to supersymmetric conical defects which approach the extremal BTZ black hole for $N$ large.

The twisted sector states provide a set of excitations confined to the orbifold singularity. There is a macroscopic number of such excitations when $N$ is large, and it will be interesting to see to what extent these excitations mimic the behavior of the black hole states that appear at higher energy. Work in this direction is in progress. 
Acknowledgments: We thank V. Balasubramanian, B. da Cunha, J. de Boer, J. Maldacena, A. Giveon, E. Keski-Vakkuri, D. Kutasov and S. Ross for discussions and correspondence. This work was supported by DOE grant DE-FG02-90ER40560 . 


\section{References}

[1] O. Aharony, S. S. Gubser, J. Maldacena, H. Ooguri, and Y. Oz, "Large n field theories, string theory and gravity," Phys. Rept. 323 (2000) 183-386, hep-th/9905111.

[2] L. Susskind and E. Witten, "The holographic bound in anti-de sitter space," hep-th/9805114.

[3] T. Banks, M. R. Douglas, G. T. Horowitz, and E. Martinec, "Ads dynamics from conformal field theory," hep-th/9808016.

[4] V. Balasubramanian, P. Kraus, A. Lawrence, and S. P. Trivedi, "Holographic probes of anti-de sitter space-times," Phys. Rev. D59 (1999) 104021, hep-th/9808017.

[5] A. W. Peet and J. Polchinski, "Uv/ir relations in ads dynamics," Phys. Rev. D59 (1999) 065011, hep-th/9809022.

[6] G. T. Horowitz and N. Itzhaki, "Black holes, shock waves, and causality in the ads/cft correspondence," JHEP 02 (1999) 010, hep-th/9901012.

[7] J. Polchinski, L. Susskind, and N. Toumbas, "Negative energy, superluminosity and holography," Phys. Rev. D60 (1999) 084006, hep-th/9903228.

[8] A. Giveon, D. Kutasov, and N. Seiberg, "Comments on string theory on $\operatorname{ads}(3), "$ Adv. Theor. Math. Phys. 2 (1998) 733-780, hep-th/9806194.

[9] E. Martinec and V. Sahakian, "Black holes and five-brane thermodynamics," Phys. Rev. D60 (1999) 064002, hep-th/9901135.

[10] M. Banados, C. Teitelboim, and J. Zanelli, "The black hole in three-dimensional space-time," Phys. Rev. Lett. 69 (1992) 1849-1851, hep-th/9204099.

[11] M. Banados, M. Henneaux, C. Teitelboim, and J. Zanelli, "Geometry of the (2+1) black hole," Phys. Rev. D48 (1993) 1506-1525, gr-qc/9302012.

[12] O. Coussaert and M. Henneaux, "Supersymmetry of the $(2+1)$ black holes," Phys. Rev. Lett. 72 (1994) 183-186, http://arXiv.org/abs/hep-th/9310194.

[13] J. M. Izquierdo and P. K. Townsend, "Supersymmetric space-times in $(2+1)$ ads supergravity models," Class. Quant. Grav. 12 (1995) 895-924, http://arXiv.org/abs/gr-qc/9501018. 
[14] M. Cvetic and F. Larsen, "Near horizon geometry of rotating black holes in five dimensions," Nucl. Phys. B531 (1998) 239-255, hep-th/9805097.

[15] J. R. David, G. Mandal, S. Vaidya, and S. R. Wadia, "Point mass geometries, spectral flow and ads(3)-cft(2) correspondence," Nucl. Phys. B564 (2000) 128-141, hep-th/9906112.

[16] V. Balasubramanian, J. de Boer, E. Keski-Vakkuri, and S. F. Ross, "Supersymmetric conical defects: Towards a string theoretic description of black hole formation," hep-th/0011217.

[17] J. Maldacena and L. Maoz, "De-singularization by rotation," hep-th/0012025.

[18] S. D. Mathur, "Gravity on $\operatorname{ads}(3)$ and flat connections in the boundary cft," http://arXiv.org/abs/hep-th/0101118.

[19] O. Lunin and S. D. Mathur, "Metric of the multiply wound rotating string," Nucl. Phys. B610 (2001) 49-76, http://arXiv.org/abs/hep-th/0105136.

[20] A. Strominger and C. Vafa, "Microscopic origin of the bekenstein-hawking entropy," Phys. Lett. B379 (1996) 99-104, hep-th/9601029.

[21] J. D. Brown and M. Henneaux, "Central charges in the canonical realization of asymptotic symmetries: An example from three-dimensional gravity," Commun. Math. Phys. 104 (1986) 207-226.

[22] A. Strominger, "Black hole entropy from near-horizon microstates," JHEP 02 (1998) 009, hep-th/9712251.

[23] A. Sen, "Tachyon condensation on the brane antibrane system," JHEP 08 (1998) 012, hep-th/9805170.

[24] E. Witten, "Instability of the kaluza-klein vacuum," Nucl. Phys. B195 (1982) 481.

[25] G. T. Horowitz and R. C. Myers, "The ads/cft correspondence and a new positive energy conjecture for general relativity," Phys. Rev. D59 (1999) 026005, hep-th/9808079.

[26] N. Seiberg and E. Witten, "The d1/d5 system and singular cft," JHEP 04 (1999) 017, hep-th/9903224.

[27] F. Larsen and E. Martinec, "U(1) charges and moduli in the d1-d5 system," JHEP 06 (1999) 019, hep-th/9905064.

[28] D. Gepner and E. Witten, "String theory on group manifolds," Nucl. Phys. B278 (1986) 493. 
[29] J. Polchinski, "String theory, vol. 1,".

[30] L. Dixon, M. Peskin, and J. Lykken, "N=2 superconformal symmetry and so(2,1) current algebra," Nucl. Phys. B325 (1989) 329-355.

[31] J. Maldacena and H. Ooguri, "Strings in ads(3) and sl(2,r) wzw model. i," hep-th/0001053.

[32] L. Dixon, D. Friedan, E. Martinec, and S. Shenker, "The conformal field theory of orbifolds," Nucl. Phys. B282 (1987) 13-73.

[33] R. Argurio, A. Giveon, and A. Shomer, "Superstrings on ads(3) and symmetric products," JHEP 12 (2000) 003, hep-th/0009242.

[34] D. Gepner and Z. an Qiu, "Modular invariant partition functions for parafermionic field theories," Nucl. Phys. B285 (1987) 423.

[35] D. Kutasov, F. Larsen, and R. G. Leigh, "String theory in magnetic monopole backgrounds," Nucl. Phys. B550 (1999) 183-213, hep-th/9812027.

[36] P. D. Vecchia, V. G. Knizhnik, J. L. Petersen, and P. Rossi, "A supersymmetric wess-zumino lagrangian in two-dimensions," Nucl. Phys. B253 (1985) 701.

[37] D. Friedan, E. Martinec, and S. Shenker, "Conformal invariance, supersymmetry and string theory," Nucl. Phys. B271 (1986) 93.

[38] M. R. Douglas and G. Moore, "D-branes, quivers, and ale instantons," hep-th/9603167.

[39] E. G. Gimon and C. V. Johnson, "K3 orientifolds," Nucl. Phys. B477 (1996) $715-745$, hep-th/9604129.

[40] D. Berenstein, R. Corrado, and J. Distler, "Aspects of ale matrix models and twisted matrix strings," Phys. Rev. D58 (1998) 026005, hep-th/9712049.

[41] D.-E. Diaconescu, M. R. Douglas, and J. Gomis, "Fractional branes and wrapped branes," JHEP 02 (1998) 013, hep-th/9712230.

[42] J. Maldacena, G. Moore, and N. Seiberg, "Geometrical interpretation of d-branes in gauged wzw models," hep-th/0105038.

[43] P. S. Aspinwall, "Resolution of orbifold singularities in string theory," hep-th/9403123.

[44] D. Anselmi, M. Billo, P. Fre, L. Girardello, and A. Zaffaroni, "Ale manifolds and conformal field theories," Int. J. Mod. Phys. A9 (1994) 3007-3058, hep-th/9304135. 
[45] T. Eguchi and A. Taormina, "Unitary representations of $\mathrm{n}=4$ superconformal algebra," Phys. Lett. B196 (1987) 75.

[46] A. Schwimmer and N. Seiberg, "Comments on the $n=2, n=3, n=4$ superconformal algebras in two-dimensions," Phys. Lett. B184 (1987) 191.

[47] W. Nahm and K. Wendland, "A hiker's guide to k3: Aspects of $\mathrm{n}=(4,4)$ superconformal field theory with central charge c $=6$," hep-th/9912067.

[48] H. Ooguri and C. Vafa, "Two-dimensional black hole and singularities of cy manifolds," Nucl. Phys. B463 (1996) 55-72, hep-th/9511164.

[49] E. Witten, "On string theory and black holes," Phys. Rev. D44 (1991) 314-324.

[50] G. Mandal, A. M. Sengupta, and S. R. Wadia, "Classical solutions of two-dimensional string theory," Mod. Phys. Lett. A6 (1991) 1685-1692.

[51] E. J. Martinec, "Algebraic geometry and effective lagrangians," Phys. Lett. B217 (1989) 431.

[52] C. Vafa and N. Warner, "Catastrophes and the classification of conformal theories," Phys. Lett. B218 (1989) 51.

[53] A. Giveon and D. Kutasov, "Little string theory in a double scaling limit," JHEP 10 (1999) 034, hep-th/9909110.

[54] A. Giveon and D. Kutasov, "Comments on double scaled little string theory," JHEP 01 (2000) 023, hep-th/9911039.

[55] V. A. Fateev and A. B. Zamolodchikov, "Parafermionic currents in the two-dimensional conformal quantum field theory and selfdual critical points in z(n) invariant statistical systems," Sov. Phys. JETP 62 (1985) 215-225.

[56] J. Maldacena, "The large $\mathrm{n}$ limit of superconformal field theories and supergravity," Adv. Theor. Math. Phys. 2 (1998) 231-252, hep-th/9711200.

[57] J. de Boer, H. Ooguri, H. Robins, and J. Tannenhauser, "String theory on ads(3)," JHEP 12 (1998) 026, hep-th/9812046.

[58] D. Kutasov and N. Seiberg, "More comments on string theory on ads(3)," JHEP 04 (1999) 008, hep-th/9903219.

[59] M. Henneaux, L. Maoz, and A. Schwimmer, "Asymptotic dynamics and asymptotic symmetries of three-dimensional extended ads supergravity," Annals Phys. 282 (2000) 31-66, http://arXiv.org/abs/hep-th/9910013. 